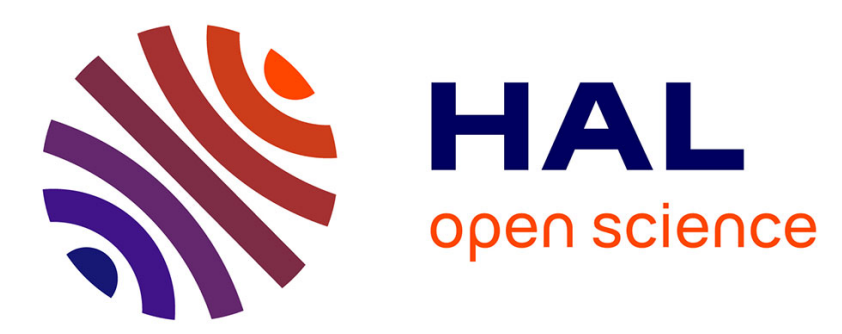

\title{
Effect of saddle point anisotropy of point defects on their absorption by dislocations and cavities
}

\author{
Carpentier D., T. Jourdan, Y. Le Bouar, M.-C. Marinica
}

\section{To cite this version:}

Carpentier D., T. Jourdan, Y. Le Bouar, M.-C. Marinica. Effect of saddle point anisotropy of point defects on their absorption by dislocations and cavities. Acta Materialia, 2017, 136, pp.323-334. 10.1016/j.actamat.2017.07.013 . hal-01632426

\section{HAL Id: hal-01632426 \\ https://hal.science/hal-01632426}

Submitted on 5 Jul 2021

HAL is a multi-disciplinary open access archive for the deposit and dissemination of scientific research documents, whether they are published or not. The documents may come from teaching and research institutions in France or abroad, or from public or private research centers.
L'archive ouverte pluridisciplinaire HAL, est destinée au dépôt et à la diffusion de documents scientifiques de niveau recherche, publiés ou non, émanant des établissements d'enseignement et de recherche français ou étrangers, des laboratoires publics ou privés. 


\title{
Effect of saddle point anisotropy of point defects on their absorption by dislocations and cavities
}

\author{
D. Carpentier ${ }^{\mathrm{a}}$, T. Jourdan ${ }^{\mathrm{a}, *}$, Y. Le Bouar ${ }^{\mathrm{b}}$, M.-C. Marinica ${ }^{\mathrm{a}}$ \\ ${ }^{a}$ DEN-Service de Recherches de Métallurgie Physique, CEA, Université Paris-Saclay, \\ F-91191, Gif-sur-Yvette, France \\ ${ }^{b}$ LEM, CNRS/ONERA, 29 av. de la division Leclerc, 92322 Châtillon, France
}

\begin{abstract}
Developing predictive models for the microstructure evolution of materials requires an accurate description of the point defects fluxes to the different sinks, such as dislocations, grain boundaries and cavities. This work aims at improving the evaluation of sink strengths of dislocations and cavities using object kinetic Monte-Carlo simulations parametrized with density functional theory calculations. The present accurate description of point defects migration enables quantitative assessment of the influence of the point defects anisotropy at saddle point. The results in aluminum show that the anisotropy at saddle point has a large influence on sink strengths. In particular, this anisotropy leads to the cavity being a biased sink. These results are explained by the analysis of the point defect trajectories to the sinks, which are shown to be strongly affected by the saddle point anisotropy.
\end{abstract}

Keywords: kinetic Monte Carlo, Diffusion, Irradiation, Dislocation, Cavity

\section{Introduction}

Supersaturation of point defects in metals occurs, for example, after plastic deformation [1, hydrogen charging [2, 3, solid or liquid state quenching [4, 5, 6] and irradiation [7. This supersaturation leads to the formation of vacancy

\footnotetext{
* Corresponding author

Email address: thomas.jourdan@cea.fr (T. Jourdan)
} 
clusters such as voids, stacking fault tetrahedra and dislocation loops and, in the case of irradiation, self-interstitial clusters. These clusters are sinks for point defects and grow, thereby affecting the macroscopic properties of materials.

Fluxes of point defects to the sinks of the microstructure strongly depend on the elastic field created by the sinks, which modify the energy landscape. Striking examples of the role of elastic interactions between sinks and point defects on the final microstructure come from materials under irradiation. Swelling [7] and irradiation creep [8, 9] are due to a slight imbalance of absorption of point defects (self-interstitial atoms (SIAs) and vacancies) by the different sinks of the microstructure. In the so-called "dislocation bias model", swelling is due to the preferential absorption of interstitials by dislocations, which is responsible for a net flux of vacancies to cavities [10]. In this model, cavities are assumed to be neutral sinks, which means that they have no absorption bias for interstitials. Some models for irradiation creep, such as SIPA (Stress Induced Preferred Absorption), rely on the difference in climb velocity of different dislocation populations, depending on their orientation with respect to the applied stress. In such models, the climb velocity depends not only on the applied stress, but also on the stress field created by the dislocations [8]. In order to simulate irradiation induced phenomena such as swelling and creep, a proper description of the effect of the elastic field created by sinks on point defect migration is therefore crucial.

The simulation of long term microstructures under irradiation is conveniently performed by rate equation cluster dynamics [11, 12, 13, 14. In this kind of mean-field model, the variation of the migration energy along the point defect trajectory cannot be taken into account explicitly. The effect of elastic interactions on point defect diffusion is found in the sink strengths for SIAs $\left(k_{i}^{2}\right)$ and vacancies $\left(k_{v}^{2}\right)$. The bias, which is defined as the relative difference between the sink strengths $k_{i}^{2}$ and $k_{v}^{2}$, quantifies to what extent a sink preferentially absorbs SIAs or vacancies, depending on its sign.

A large amount of data exists about sink strengths and bias values, but the scattering is rather high. Assuming that dislocations are the only biased 
sinks, the dislocation bias can be inferred from experimental swelling data, using standard rate theory models [15, 16, 17, 18, 19. However, swelling is a combination of the bias and the fraction of freely migrating defects, which is not precisely known for ion and neutron irradiations. Therefore, there is some uncertainty about the bias values obtained by such methods. Depending on the experimental data and the assumptions of the rate theory model, the dislocation bias values typically range between 0.01 and 0.35 . Sink strengths and biases can also be computed. A common way is to solve the drift-diffusion equation for the concentration of point defects around a sink. This method was used for the first calculations, mostly for simple geometries and simple description of the sinkpoint defect interaction [20, 21, 22, 23]. For more complex cases, phase field [24] and object kinetic Monte Carlo (OKMC) simulations have been used [25, 26, 27]. OKMC methods are particularly handy to take into account the effect of elastic interactions at stable and saddle points [28, 29.

The influence of elastic interactions at saddle position on the value of sink strength was emphasized by Dederichs and Schroeder [30. Although this effect had been discussed previously [31, 32, 33], these authors also suggested that the anisotropy of point defects at saddle position could have an effect on the sink strength. Such an effect was later confirmed for straight dislocations [34, 35, 36, 37, infinitesimal dislocation loops [38] and voids [39]. However, all these works contain approximations to make calculations tractable, so the values of sink strengths significantly vary from one study to the other [37]. In addition, the elastic dipoles of point defects are based on empirical potential calculations, which are not always in agreement with first-principles calculations. Only recently, the effect of saddle point anisotropy has been shown for the sink strength of semi-coherent interfaces, using OKMC simulations parameterized with density functional theory (DFT) calculations [29.

In the present work, we perform OKMC simulations to study the sink strengths of straight dislocations and spherical cavities in pure aluminum, in order to assess the role of saddle point anisotropy. To that purpose, the elastic interactions between sinks and point defects are modeled explicitly in the 
OKMC code. Point defects are represented by their elastic dipole tensors computed by DFT calculations.

This paper is structured as follows. Section 2 describes the method used to calculate sink strengths. Section 3 presents the study of the straight dislocation. The case of a spherical cavity is treated in section 4

\section{Methods}

\subsection{OKMC simulations and sink strength calculation}

Sink strengths are calculated with an OKMC code [29, allowing the simulation of many point defect trajectories in an efficient way, and making it possible to account for the point defects properties and elastic interactions between point defects and sinks $[28,40,29]$. A single type of sink is introduced in the simulation box. It can be a dislocation or a spherical cavity. The sinks are considered as immobile and remain unchanged after absorption of defects. The temperature is set to $300 \mathrm{~K}$. At this temperature, thermal equilibrium concentration of point defects is far smaller than the concentration imposed by irradiation. Therefore, thermal generation of point defects by the sinks is neglected.

SIAs and vacancies are considered separately, in dedicated simulations, thus no recombination is possible. They are generated uniformly at a constant creation rate $G_{0}$ (in $s^{-1}$ ), and migrate inside the box by performing atomic jumps until they are absorbed by the sink. The migrating point defects do not react with each other to form clusters and no long-range interactions between point defects are considered. Periodic boundary conditions are used in all 3 dimensions. Point defects are considered as absorbed by the sink when the distance $d$ between the sink center and the point defect verifies $d \leq d_{\text {reac }}$ where $d_{\text {reac }}$ is the reaction distance depending on the nature of the sink.

To increment the simulation time, a residence time algorithm is used 41, 42. At a given time $t$, the time step is given by $\Delta t=-\ln \left(r_{1}\right) / \Gamma_{\text {tot }}$ where $r_{1}$ is a random number chosen in $] 0,1]$ and $\Gamma_{\text {tot }}$ is the sum of the frequencies of all $N_{\mathrm{e}}$ possible events, $i$. e. $\Gamma_{\text {tot }}=\sum_{i=0}^{N_{\mathrm{e}}-1} \Gamma_{i}$. The possible events are the creation 
of a point defect due to irradiation (frequency $\Gamma_{0}=G_{0}$ ) or an atomic jump from a stable position to a neighboring one (frequency $\Gamma_{i}, i=1, \ldots, N_{\mathrm{e}}-$ 1). The chosen event $j$ is such that $\sum_{i=0}^{j-1} \Gamma_{i}<r_{2} \Gamma_{\text {tot }} \leq \sum_{i=0}^{j} \Gamma_{i}$, with $r_{2}$ a random number chosen in ]0,1]. Frequencies of atomic jumps are given by $\Gamma_{i}=\nu_{0} \exp \left(-\Delta E_{i} /\left(k_{\mathrm{B}} T\right)\right)$, with $\nu_{0}$ the attempt frequency assumed to be the same for all jumps, $k_{\mathrm{B}}$ is the Boltzmann constant, $T$ the temperature and $\Delta E_{i}=$ $E_{i}^{\text {sad }}-E_{i}^{\text {sta }}$ the difference of energy between the saddle point of the jump and the initial stable position.

The energy of point defects at stable point $E_{i}^{\text {sta }}$ and at saddle point $E_{i}^{\text {sad }}$ are given by

$$
\begin{aligned}
E_{i}^{\mathrm{sta}} & =-\sum_{j, k} P_{i, j k}^{\mathrm{sta}} \epsilon_{j k}\left(\boldsymbol{r}_{i}^{\mathrm{sta}}\right) \\
E_{i}^{\mathrm{sad}} & =E^{\mathrm{m}}-\sum_{j, k} P_{i, j k}^{\mathrm{sad}} \epsilon_{j k}\left(\boldsymbol{r}_{i}^{\mathrm{sad}}\right),
\end{aligned}
$$

where $E^{\mathrm{m}}$ is the migration energy without elastic interactions and $\boldsymbol{P}^{\text {sta }}$ and $\boldsymbol{P}^{\text {sad }}$ are elastic dipole tensors ( $\mathbf{P}$-tensors) describing the point defects at stable and saddle positions, respectively [43, 44]. The saddle position $\boldsymbol{r}^{\text {sad }}$ is simply considered as the midpoint along the reaction coordinate between the two stable positions involved in the jump. The strain, written $\boldsymbol{\epsilon}$, is generated by the sink. Since we focus on the effect of saddle point anisotropy, we consider that $\mathbf{P}$-tensors do not depend on the local strain, $i$. $e$. we neglect polarisability effects 43 . Other energy terms, such that higher order terms in the multipole expansion [44] and image interactions for voids [45, 46], are also neglected.

In our simulations, unless otherwise specified, the calculations are performed in isotropic elasticity, using available analytical expressions of the strain produced by the sink. This assumption is expected to be reasonable in the case of face centered cubic (FCC) aluminum, studied here, because the elastic moduli tensor of this material is only weakly anisotropic (see Tab. 1). This makes it possible to focus on the effect of point defects anisotropy only. However, to check the influence of the anisotropy of the elastic moduli tensor on the sink strength, simulations are also performed in anisotropic elasticity, using the values of elas- 
tic constants given in [4]. In that case, a Fast Fourier Transform (FFT)-based method is used to compute the strain field at mechanical equilibrium [48, 49, 50,

\begin{tabular}{|c|c|c|c|}
\hline \multicolumn{3}{|c|}{ Elastic moduli tensor terms and Zener anisotropy ratio [4] } \\
\hline$C_{11}(\mathrm{GPa})$ & $C_{12}(\mathrm{GPa})$ & $C_{44}(\mathrm{GPa})$ & $A=2 C_{44} /\left(C_{11}-C_{12}\right)$ \\
\hline 106.51 & 60.38 & 27.8 & 1.21 \\
\hline \multicolumn{3}{|c|}{ Constants for isotropic elasticity } \\
\hline \multicolumn{2}{|c|}{ Poisson's ratio $\nu$} & \multicolumn{2}{c|}{ Shear modulus $\mu(\mathrm{GPa})$} \\
\hline \multicolumn{2}{|c|}{0.35} & \multicolumn{2}{c|}{25.91} \\
\hline
\end{tabular}

Table 1: Elastic constants of aluminum at $300 \mathrm{~K}$. The isotropic elastic constants are calculated from the tensor terms by the Voigt average [51].

In the mean field rate theory, the sink strength $k^{2}$ defines the ability of a sink to absorb point defects. In this formalism, the evolution equation of the average number of defects $\bar{N}$ is given by

$$
\frac{\mathrm{d} \bar{N}}{\mathrm{~d} t}=G_{0}-k^{2} D \bar{N},
$$

with $D$ the point defect diffusion coefficient, which reads

$$
D=\alpha a_{0}^{2} \nu_{0} \exp \left(-\frac{E^{\mathrm{m}}}{k_{\mathrm{B}} T}\right) .
$$

In this equation, $\alpha=\frac{2}{3}$ for SIAs ( $\langle 100\rangle$ dumbbells) and $\alpha=1$ for vacancies, and $a_{0}$ is the lattice parameter of the FCC lattice.

The absorption of point defects by the sink is expressed in Eq. (3) through the second term in the right-hand side. When the steady state is reached, the sink strength can be computed by

$$
k^{2}=\frac{G_{0}}{D \bar{N}} .
$$

Two sink strength values are obtained, $k_{\text {SIA }}^{2}$ for SIAs and $k_{\text {vac }}^{2}$ for vacancies. From these two values, the sink bias $B$ can be calculated by [52]

$$
B=\frac{k_{\mathrm{SIA}}^{2}-k_{\mathrm{vac}}^{2}}{k_{\mathrm{SIA}}^{2}} .
$$


This parameter describes the ability of the sink to absorb more SIAs than vacancies.

In the OKMC simulations, the number of point defects in the box $N$ is recorded every $10^{4}$ steps. At the end of the simulation, the average number of defects at steady state $\bar{N}$ is computed by averaging the recorded values. The sink strengths and bias values are then computed using Eqs. (5)- (6). The simulated times are chosen to obtain sufficient accuracy on the value of $\bar{N}$, and thus on $k^{2}$. A block-averaging procedure [53] is used to compute the error estimates $\sigma$ on the sink strength and bias. In the following, the error bars in figures correspond to $\pm 3 \sigma$.

\subsection{Representation of point defects}

To compute interactions between the sink and the point defects, the $\mathbf{P}$ tensors at stable points $\boldsymbol{P}^{\text {sta }}$ and at saddle points $\boldsymbol{P}^{\text {sad }}$ are needed. The values of $\mathbf{P}$-tensors for SIAs and vacancies in aluminum are computed by DFT calculations. The DFT simulation cell for SIA/vacancy contains $256 \pm 1$ atoms. Calculations are performed with VASP code using the projector augmented wave framework [54, 55, 56, 57. The plane wave energy cutoff is set to $450 \mathrm{eV}$ and the Hermite-Gaussian broadening-width for Brillouin zone integration is $0.2 \mathrm{eV}$. The calculations are performed including the s semi-core states $[\mathrm{Ne}] 3 \mathrm{~s}^{2} 3 \mathrm{p}^{1}$. The exchange correlation energy is evaluated using the Perdew-Burke-Ernzerhof (PBE) Generalized Gradient Approximation (GGA) [58]. The $k$-point grid mesh is set to shifted $3 \times 3 \times 3$ grid. Each configuration is relaxed using the gradient conjugate technique. The climbing image method [59] using 15 images is used in order to localize the saddle point. In relaxed configurations, minimum or saddle point, the forces on each atom are lower than $0.001 \mathrm{eV} / \AA$. With such settings the relative error in the energy and $\mathbf{P}$-tensor components is estimated to be less than $0.5 \%$ and $4 \%$, respectively, from converged values [29].

For the configurations illustrated in Tab.2, the following results are obtained:

1. For SIAs, migration energy of $E^{\mathrm{m}}=0.105 \mathrm{eV}$, and

- at stable point, dumbbell along [100]: 


$$
\boldsymbol{P}_{\text {SIA }, \text { ref }}^{\text {sta }}=\left[\begin{array}{ccc}
19.652 & 0 & 0 \\
0 & 18.518 & 0 \\
0 & 0 & 18.518
\end{array}\right] \mathrm{eV}
$$

- at saddle point, for a [100]-to-[010] migration path:

$$
\boldsymbol{P}_{\text {SIA,ref }}^{\mathrm{sad}}=\left[\begin{array}{ccc}
19.498 & 1.133 & 0 \\
1.133 & 19.498 & 0 \\
0 & 0 & 19.034
\end{array}\right] \mathrm{eV}
$$

2. For vacancies, migration energy of $E^{\mathrm{m}}=0.605 \mathrm{eV}$ and

- at stable point:

$$
\boldsymbol{P}_{\text {vac,ref }}^{\text {sta }}=\left[\begin{array}{ccc}
-3.238 & 0 & 0 \\
0 & -3.238 & 0 \\
0 & 0 & -3.238
\end{array}\right] \mathrm{eV}
$$

- at saddle point, for a migration along the [110] direction:

$$
\boldsymbol{P}_{\mathrm{vac}, \mathrm{ref}}^{\mathrm{sad}}=\left[\begin{array}{ccc}
-2.866 & -0.080 & 0 \\
-0.080 & -2.866 & 0 \\
0 & 0 & 1.000
\end{array}\right] \mathrm{eV}
$$

These values show that SIAs are weakly anisotropic at stable and saddle points, while vacancies are perfectly isotropic at stable point and strongly anisotropic at saddle point. The anisotropy of SIAs at stable point is in excellent agreement with the experimental value $\left|P_{11}-P_{22}\right|=1.1 \pm 0.3 \mathrm{eV}[60$. The relaxation volumes at stable point for interstitials and vacancies, deduced from the trace of the dipole tensor through $\Delta V^{\text {sta }}=\operatorname{Tr}(\boldsymbol{P}) / 3 K$ with $K$ the bulk modulus, seem slightly overestimated: they are equal to $-0.40 \Omega$ for vacancies and $2.35 \Omega$ for interstitials ( $\Omega$ is the atomic volume), while experimental values range from -0.05 to $-0.38 \Omega$ for vacancies and are equal to $1.9 \pm 0.4 \Omega$ for interstitials 61, 62]. 


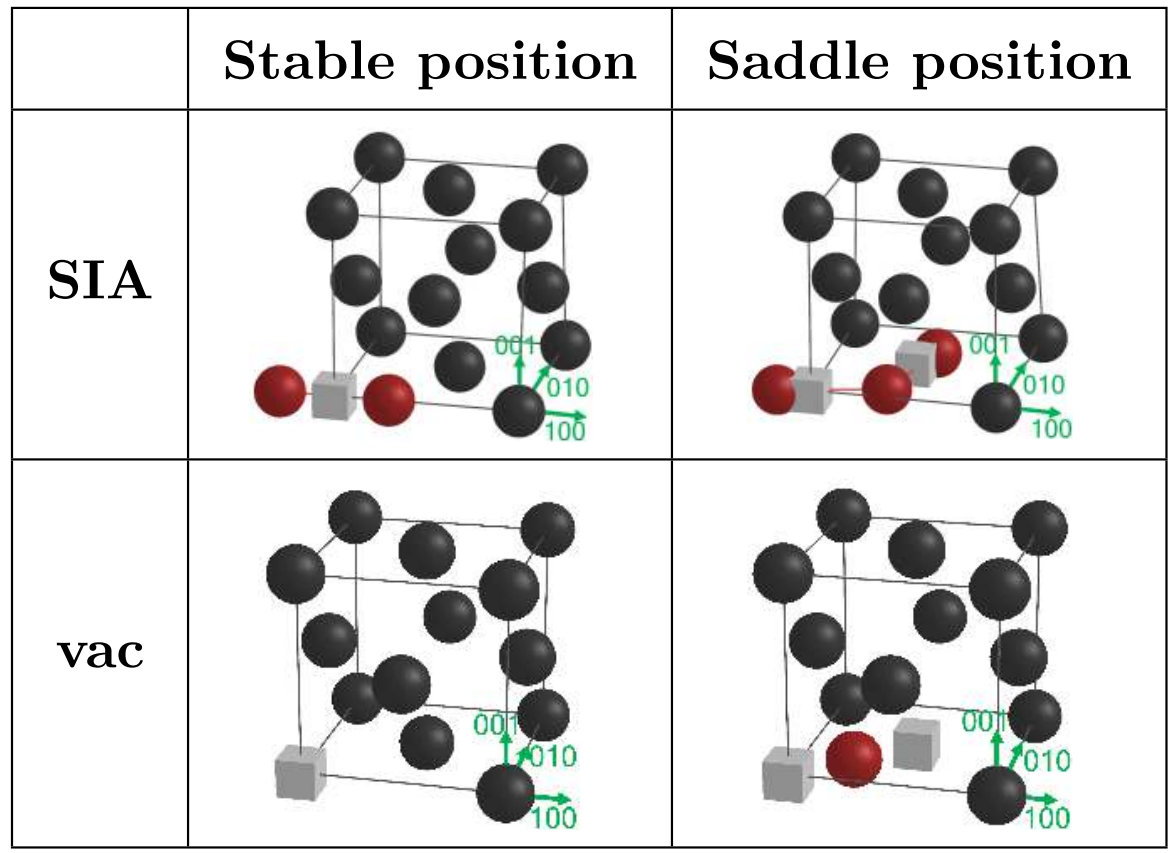

Table 2: Schematic representations of stable and saddle positions of point defects in FCC lattice, with orientations corresponding to the P-tensors in Eqs. 7 to 10 . Here, black spheres represent the regular lattice atomic positions, red spheres represent atomic positions outside of lattice nodes and gray cubes represent vacancies.

To understand which properties of point defects have a major influence on the sink strengths, different simulation cases are built, using the previous $\mathbf{P}$ tensors or simplified versions of them. The cases are given in Tab. 3 .

The first case (case $0, \boldsymbol{P}_{0}^{\text {sta }}=\mathbf{0}$ and $\boldsymbol{P}_{0}^{\text {sad }}=\mathbf{0}$ ) is considered as a reference to validate the method used in this work, by comparing the calculated sink strengths to the corresponding analytical solutions. Indeed, for the boundary conditions considered here, the analytical expressions only exist when the sink strain field in not considered, $i$. e. when the point defects do not interact with the sink.

An accurate assessment of the effects of elastic interactions on the sink strength is done using the real point defect description (case $2, \boldsymbol{P}_{2}^{\text {sta }}=\boldsymbol{P}_{\text {ref }}^{\text {sta }}$ and $\boldsymbol{P}_{2}^{\mathrm{sad}}=\boldsymbol{P}_{\mathrm{ref}}^{\mathrm{sad}}$ ). 
The following two approximations $2^{\prime}$ and $2^{\prime \prime}$ are used to identify the point defect properties influencing the sink strengths. Case $2^{\prime}$ corresponds to point defects with isotropic dipole tensors at saddle point but with the same relaxation volume as for case 2 :

$$
\begin{aligned}
& \boldsymbol{P}_{2^{\prime}}^{\text {sta }}=\boldsymbol{P}_{\text {ref }}^{\text {sta }} \\
& \boldsymbol{P}_{2^{\prime}}^{\text {sad }}=\frac{1}{3} \operatorname{Tr}\left(\boldsymbol{P}_{\mathrm{ref}}^{\mathrm{sad}}\right) \boldsymbol{I},
\end{aligned}
$$

where $\boldsymbol{I}$ is the identity tensor. Such an approximation permits to enlighten the effect of saddle point anisotropy by comparing results to case 2 . For case $2^{\prime \prime}$, a further approximation is used: dipole tensors are taken isotropic at stable point, with the same relaxation volume as cases 2 and $2^{\prime}$ :

$$
\begin{aligned}
\boldsymbol{P}_{2^{\prime \prime}}^{\mathrm{sta}} & =\frac{1}{3} \operatorname{Tr}\left(\boldsymbol{P}_{\mathrm{ref}}^{\mathrm{sta}}\right) \boldsymbol{I} \\
\boldsymbol{P}_{2^{\prime \prime}}^{\mathrm{sad}} & =\frac{1}{3} \operatorname{Tr}\left(\boldsymbol{P}_{\mathrm{ref}}^{\mathrm{sad}}\right) \boldsymbol{I} .
\end{aligned}
$$

Therefore, by comparing cases $2^{\prime}$ and $2^{\prime \prime}$, the effect of anisotropy at stable point can be evaluated.

Finally, a traditional approximation in the calculation of sink strengths consists in ignoring the differences in point defect properties at stable and saddle points. Defect properties are given solely by the relaxation volume at stable point $\Delta V^{\text {sta }}[52,63,22,64,23]$. This is tantamount to using the following dipole tensors (case 1):

$$
\boldsymbol{P}_{1}^{\text {sta }}=\boldsymbol{P}_{1}^{\text {sad }}=\frac{1}{3} \operatorname{Tr}\left(\boldsymbol{P}_{\text {ref }}^{\text {sta }}\right) \boldsymbol{I}
$$

\section{Straight dislocation}

\subsection{Case definition}

The simulation box contains two dislocations, each dislocation having the

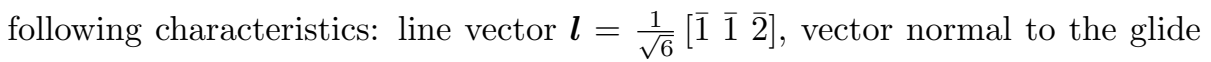
plane $\boldsymbol{n}=\frac{1}{\sqrt{3}}\left[\begin{array}{lll}1 & 1 & \overline{1}\end{array}\right]$ and Burgers vector $\boldsymbol{b}= \pm \frac{a_{0}}{2}\left[\begin{array}{lll}\overline{1} & 1 & 0\end{array}\right]\left(\|\boldsymbol{b}\|=\frac{a_{0}}{\sqrt{2}}\right)$. This 


\begin{tabular}{|c|c|c|c|c|c|c|c|c|c|}
\hline & & \multicolumn{4}{|c|}{ SIA } & \multicolumn{4}{|c|}{ vacancy } \\
\hline Case 0 & $\begin{array}{l}\text { Non interacting } \\
\text { defects }\end{array}$ & \multicolumn{4}{|c|}{$\begin{array}{l}P_{\mathrm{SIA}}^{\text {sta }}=\mathbf{0} \\
\boldsymbol{P}_{\mathrm{SIA}}^{\mathrm{sad}}=\mathbf{0}\end{array}$} & \multicolumn{4}{|c|}{$\begin{array}{l}P_{\text {vac }}^{\text {sta }}=\mathbf{0} \\
P_{\text {vac }}^{\text {sad }}=\mathbf{0}\end{array}$} \\
\hline Case 1 & $\begin{array}{l}\text { Spherical inclu- } \\
\text { sions }\end{array}$ & \multicolumn{4}{|c|}{$\begin{array}{l}\boldsymbol{P}_{\mathrm{SIA}}^{\mathrm{sta}}=18.896 \boldsymbol{I} \\
\boldsymbol{P}_{\mathrm{SIA}}^{\mathrm{sad}}=18.896 \boldsymbol{I}\end{array}$} & \multicolumn{4}{|c|}{$\begin{array}{l}\boldsymbol{P}_{\mathrm{vac}}^{\mathrm{sta}}=-3.238 \boldsymbol{I} \\
\boldsymbol{P}_{\mathrm{vac}}^{\mathrm{sad}}=-3.238 \boldsymbol{I}\end{array}$} \\
\hline Case 2 & Real defects & $P_{\mathrm{SIA}}^{\mathrm{sta}}=$ & $\begin{array}{l}{\left[\begin{array}{c}19.652 \\
0 \\
0\end{array}\right.} \\
{\left[\begin{array}{c}19.498 \\
1.133 \\
0\end{array}\right.}\end{array}$ & $\begin{array}{c}0 \\
18.518 \\
0 \\
1.133 \\
19.498 \\
0\end{array}$ & $\left.\begin{array}{c}0 \\
0 \\
18.518 \\
0 \\
0 \\
19.034\end{array}\right]$ & $\begin{array}{l}P_{\text {vac }}^{\text {sta }}= \\
P_{\text {vac }}^{\text {sad }}=\end{array}$ & $\begin{array}{l}-3.238 \boldsymbol{I} \\
{\left[\begin{array}{c}-2.866 \\
-0.080 \\
0\end{array}\right.}\end{array}$ & $\begin{array}{c}-0.080 \\
-2.866 \\
0\end{array}$ & $\left.\begin{array}{c}0 \\
0 \\
1.000\end{array}\right]$ \\
\hline Case $2^{\prime}$ & $\begin{array}{l}\text { Defects simplified } \\
\text { at saddle points }\end{array}$ & $\begin{array}{l}P_{\mathrm{SIA}}^{\mathrm{sta}}= \\
P_{\mathrm{SIA}}^{\mathrm{sad}}=\end{array}$ & \begin{tabular}{|c}
19.652 \\
0 \\
0 \\
$19.343 I$
\end{tabular} & $\begin{array}{c}0 \\
18.518 \\
0\end{array}$ & $\left.\begin{array}{c}0 \\
0 \\
18.518\end{array}\right]$ & \multicolumn{4}{|c|}{$\begin{array}{l}\boldsymbol{P}_{\mathrm{vac}}^{\mathrm{sta}}=-3.238 \boldsymbol{I} \\
\boldsymbol{P}_{\mathrm{vac}}^{\mathrm{sad}}=-1.577 \boldsymbol{I}\end{array}$} \\
\hline Case $2^{\prime \prime}$ & $\begin{array}{l}\text { Defects simplified } \\
\text { at stable and sad- } \\
\text { dle points }\end{array}$ & \multicolumn{4}{|c|}{$\begin{array}{l}\boldsymbol{P}_{\mathrm{SIA}}^{\mathrm{sad}}=18.896 \boldsymbol{I} \\
\boldsymbol{P}_{\mathrm{SIA}}^{\mathrm{sad}}=19.343 \boldsymbol{I}\end{array}$} & \multicolumn{4}{|c|}{$\begin{array}{l}\boldsymbol{P}_{\text {vac }}^{\mathrm{sad}}=-3.238 \boldsymbol{I} \\
\boldsymbol{P}_{\mathrm{vac}}^{\mathrm{sad}}=-1.577 \boldsymbol{I}\end{array}$} \\
\hline
\end{tabular}

Table 3: P-tensors for the different simulation cases. The values are given in $\mathrm{eV}$, for the configuration illustrated in Tab. 2 For vacancies, as they are isotropic at stable point, cases $2^{\prime}$ and $2^{\prime \prime}$ are identical.

corresponds to a perfect straight edge dislocation in the aluminum FCC lattice. The capture radius of the straight dislocations is set to $r_{c}=2\|\boldsymbol{b}\|$, which means that for each dislocation $d_{\text {reac }}=2\|\boldsymbol{b}\|[65,20$.

The two edge dislocations of opposite Burgers vectors are introduced in an orthorombic simulation box, according to the configuration in Fig. 1. Therefore, the dislocation density only depends on the box dimensions according to $\rho=\frac{2}{d_{x} \cdot d_{y}}$, so different densities can be studied by varying the box dimensions (keeping the aspect ratio constant). The lattice is rotated to ensure that the dislocation line vector is aligned with the $z$-direction of the box, while the Burgers vectors are aligned with the $x$-direction. Such a configuration ensures that the strain is continuous at the simulation box borders. Other configurations could have been studied, a few have been tested and the configuration choice does not alter the method nor the generality of our results.

As illustrated in Fig. 2, due to the periodic boundary conditions, the con- 
figuration is equivalent to a $2 \mathrm{D}$ array of dislocations in the $x y$-plane. To calculate the strain field, we have to sum along $x$ the strain fields of infinite $y$ columns of dislocations with alternate Burgers vectors, noted $\epsilon^{+}$and $\epsilon^{-}$for $\boldsymbol{e}_{x} \cdot \boldsymbol{b}>0$ and $\boldsymbol{e}_{x} \cdot \boldsymbol{b}<0$, respectively. The strain field generated by an infinite row of dislocations $\epsilon^{ \pm}$is given in [66] and [51]. The complete strain field is then given by summing the contributions of neighboring boxes $\boldsymbol{\epsilon}(x, y)=$ $\sum_{k=-N}^{N}\left(\epsilon^{+}\left(x+k \cdot d_{x}, y\right)+\epsilon^{-}\left(x+k \cdot d_{x}, y\right)\right)$, using a sufficiently large value for $N$ to reach convergence. We have used $N=1$, which gives a relative error on the strain lower than $10^{-3}$.

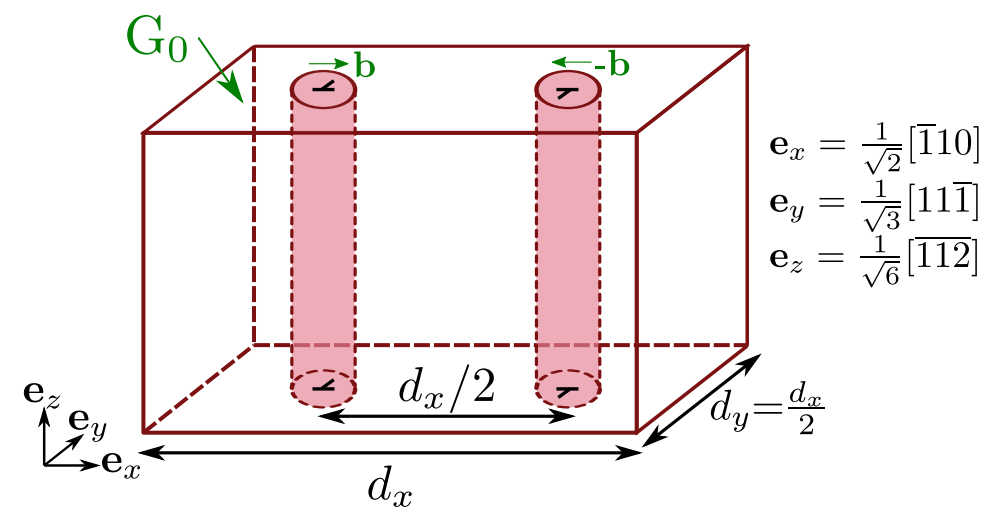

Figure 1: Configuration of the simulation box containing two edge dislocations, with opposite Burgers vectors.

\subsection{Sink strength and bias}

Values of the sink efficiency $Z=k^{2} / \rho$ and of the bias for dislocation densities from $5 \cdot 10^{13} \mathrm{~m}^{-2}$ to $10^{16} \mathrm{~m}^{-2}$, encompassing the densities in irradiated materials 67, are shown in Fig. 3 .

The reliability of our approach is proved by the good agreement between the results obtained in the case of non interacting defects (case 0, yellow squares) and the analytical expression [68. We have verified that the small differences are due to the different boundary conditions at the capture radius $r_{c}$. Indeed, the analytical treatment assumes that the concentration of point defects is zero at exactly $r=r_{c}$, while for the OKMC simulations, the defects are only removed 


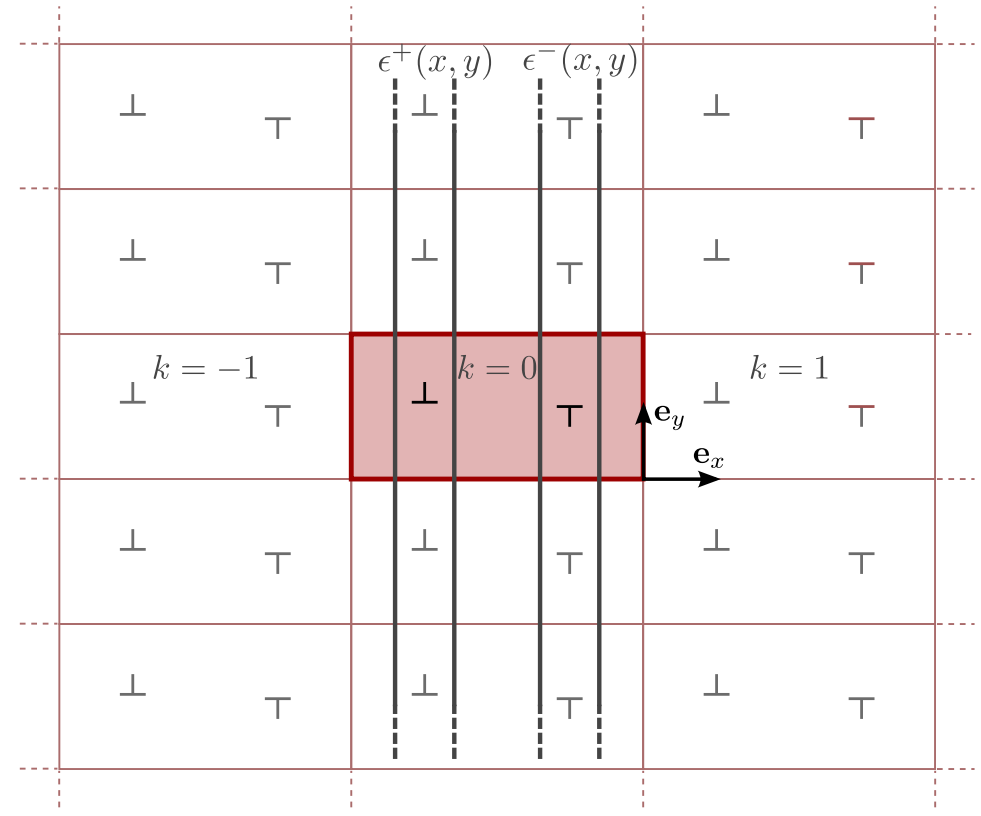

Figure 2: Schematic representation of the calculation of the strain with periodic boundary conditions. The strains $\epsilon^{+}$and $\epsilon^{-}$are generated by the columns associated to the edge dislocations with the Burgers vectors $\boldsymbol{b}$ such that $\boldsymbol{e}_{x} \cdot \boldsymbol{b}>0$ and $\boldsymbol{e}_{x} \cdot \boldsymbol{b}<0$, respectively.

after the last atomic jump that enters the capture region $r \leq r_{c}$, so the concentration is zero for some value lower than $r_{c}$. As proposed in [69, 25, 70], this difference can be accounted for by using an effective capture radius $\widetilde{r}_{c}$ slightly smaller than $r_{c}$ in the analytical formula to mimic the OKMC configuration.

When the complete $\mathbf{P}$-tensors are used (case 2 - real defects, gray downpointing triangles), sink strengths for vacancies are twice higher than the values obtained when the elastic interactions are neglected, for the highest dislocation densities sudied. This increase is even more important for SIAs than for vacancies, and the difference increases with the dislocation density. This leads to a strongly positive bias value, increasing with the dislocation density.

To identify the properties of point defects responsible for the sink strength variation, the dipole tensors are modified. By removing the anisotropy of the elastic dipole tensors at the saddle point (case $2^{\prime}$ ), the sink strength is reduced for both types of point defects. The decrease is particularly important for vacancies, 

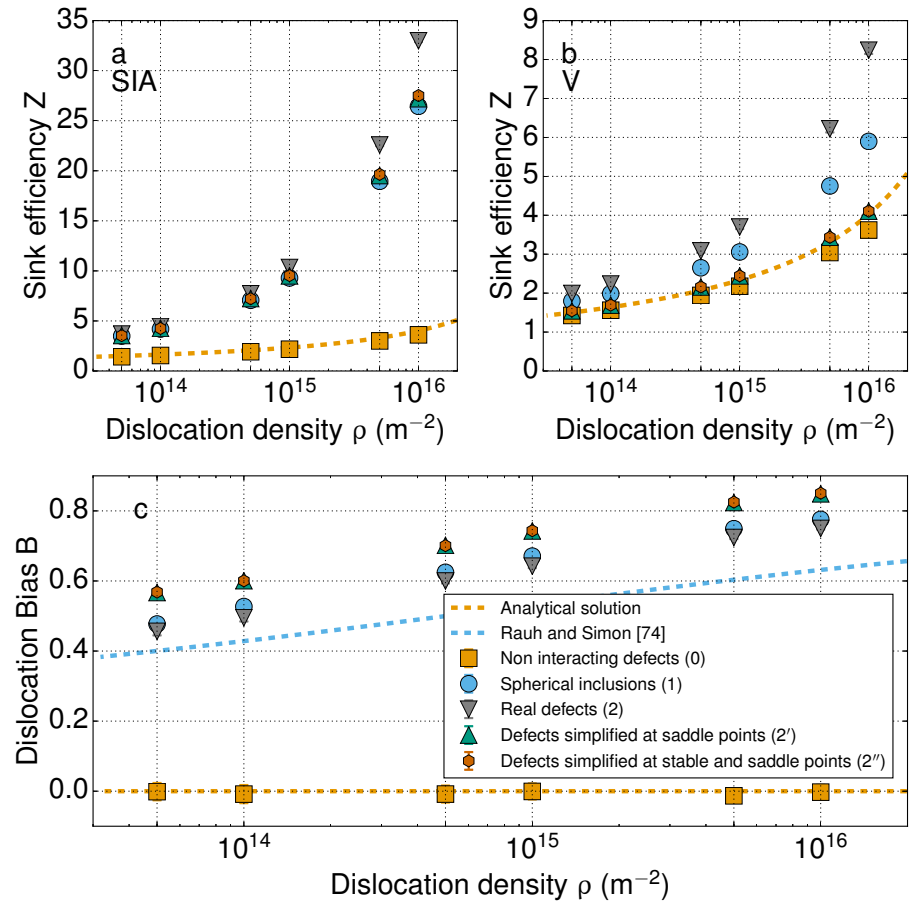

Figure 3: Straight dislocation sink efficiency for (a) SIAs and (b) vacancies, (c) bias. The error bars are displayed for all values, some of them are too small to be visible.

which are very anisotropic at saddle point. This shows that the saddle point anisotropy is responsible for a significant variation of the sink strength, through the decrease of the migration barriers near the dislocation.

The influence of stable point anisotropy can be assessed by comparing the latter results for case $2^{\prime}$ to the results for case $2^{\prime \prime}$ (defects simplified at stable and saddle points). Removing the anisotropy at stable points does not modify the sink strength values, and consequently leaves the bias unchanged. This shows that the anisotropy at stable point has no influence on the sink strength and sink bias in aluminum. This is due to the fact that, in this material, the anisotropy at stable point for SIAs is quite small, while vacancies are isotropic.

In Fig. 3 we also show the sink strength obtained when point defects have the same properties at stable and saddle points based on the relaxation volume 
at stable point (case 1, blue disks). The sink strengths are indeed quite different from the ones obtained for case 2. For SIAs, the sink strength is close to the one obtained for cases $2^{\prime}$ and $2^{\prime \prime}$, due to the fact that the relaxation volumes at saddle points are similar: for case $1, \frac{1}{3} \operatorname{Tr}\left(\boldsymbol{P}_{\text {SIA }}^{\text {sad }}\right)=18.896 \mathrm{eV}$ and for cases $2^{\prime}$ and $2^{\prime \prime}, \frac{1}{3} \operatorname{Tr}\left(\boldsymbol{P}_{\mathrm{SIA}}^{\mathrm{sad}}\right)=19.343 \mathrm{eV}$. On the contrary, the relaxation volumes at saddle points for vacancies vary a lot between case 1 and cases $2^{\prime}$ and $2^{\prime \prime}$ : for case $1, \frac{1}{3} \operatorname{Tr}\left(\boldsymbol{P}_{\mathrm{vac}}^{\mathrm{sad}}\right)=-3.238 \mathrm{eV}$ and for cases $2^{\prime}$ and $2^{\prime \prime}, \frac{1}{3} \operatorname{Tr}\left(\boldsymbol{P}_{\mathrm{vac}}^{\mathrm{sad}}\right)=$ $-1.577 \mathrm{eV}$. Consequently, the interactions are stronger for the vacancies in case 1 than in cases $2^{\prime}$ and $2^{\prime \prime}$, giving a stronger sink strength. The dislocation bias obtained in case 1 is close to the one obtained for real dipole tensors (case 2), but this is completely incidental. Finally, various analytical expressions exist in the literature for case 1, but for isolated dislocations and different boundary conditions [71, 72, 73, 74. In Fig. 3 we plot the solution provided by Rauh and Simon [74, which corresponds to the case of prescribed concentration on the system boundary (other expressions of the sink strengths correspond to slightly different boundary conditions, see Ref. 23. for a discussion of this point and a comparison of the formulas). It is shown that the results agree qualitatively but the analytical bias values are smaller than the OKMC values. Reasons for this discrepancy are given in Appendix A.

These results reveal the strong effect of elastic interactions, and more particularly show that the saddle point anisotropy of point defects is a key parameter for the variation of the sink strength and bias.

\subsection{Analysis and discussion}

In the previous section we stated that the decreasing migration barriers near the dislocation, due to the saddle point anisotropy, leads to a significant increase in the sink strengths, especially for the vacancy. These numerical results are compared with previous analytical calculations, using various approximations. These previous studies were done in copper. However, due to similar point defect properties in aluminum and copper, trends are expected to be alike in both materials. The first estimation of the effect of saddle point anisotropy 
was obtained by Skinner and Woo 36. To make the calculations tractable, they transformed the diffusion problem into a cylindrically symmetric one and assumed that the diffusion coefficient only depends on one component of the dipole tensor. They concluded that the effect was small and led to a slight increase in the sink strengths for vacancies and interstitials. With an arbitrary choice of the point defect anisotropy and diffusion tensor, Chen showed that the effect of anisotropy could be described by an effective relaxation volume [35. The increase of the relaxation volume due to the anisotropy at saddle point is such that the effect on the sink strength is rather large. Borodin and Ryazanov did not resort to some of the assumptions made by Skinner and Woo [37] and obtained a few percents decrease of the sink strength for interstitials and an increase of about $20 \%$ for vacancies. More recently, Sivak and Sivak [75] showed with OKMC calculations that the anisotropy of vacancies at saddle point is responsible for complex variations of the sink strength with the dislocation type. Here, by exactly taking into account the interaction between the dislocation and the point defect at saddle position, we show that an increase of about $20 \%$ for interstitials and $100 \%$ for vacancies can be obtained with respect to an isotropic defect.

We have also shown that the anisotropy of interstitials at stable point does not change the bias value. Several authors have pointed out that if the concentration at the boundary of the simulation cell is imposed, fluxes, and therefore sink strengths, do not depend on the stable point energy [30, 36, 37]. Therefore, in this case, no effect of defect anisotropy at stable point is expected. Here, the situation is different, since we impose the creation rate of defects and measure the sink strength by the average concentration in the simulation cell (Eq. (5)). The concentration depends on the stable point energy, so the sink strength can potentially depend on the point defect anisotropy at stable position [76]. However, the anisotropy of interstitials is so low that the difference in energy between the isotropic and the anisotropic cases is negligible, leading to no appreciable effect on the sink strength.

To have a better understanding of the influence of the saddle point anisotropy, 

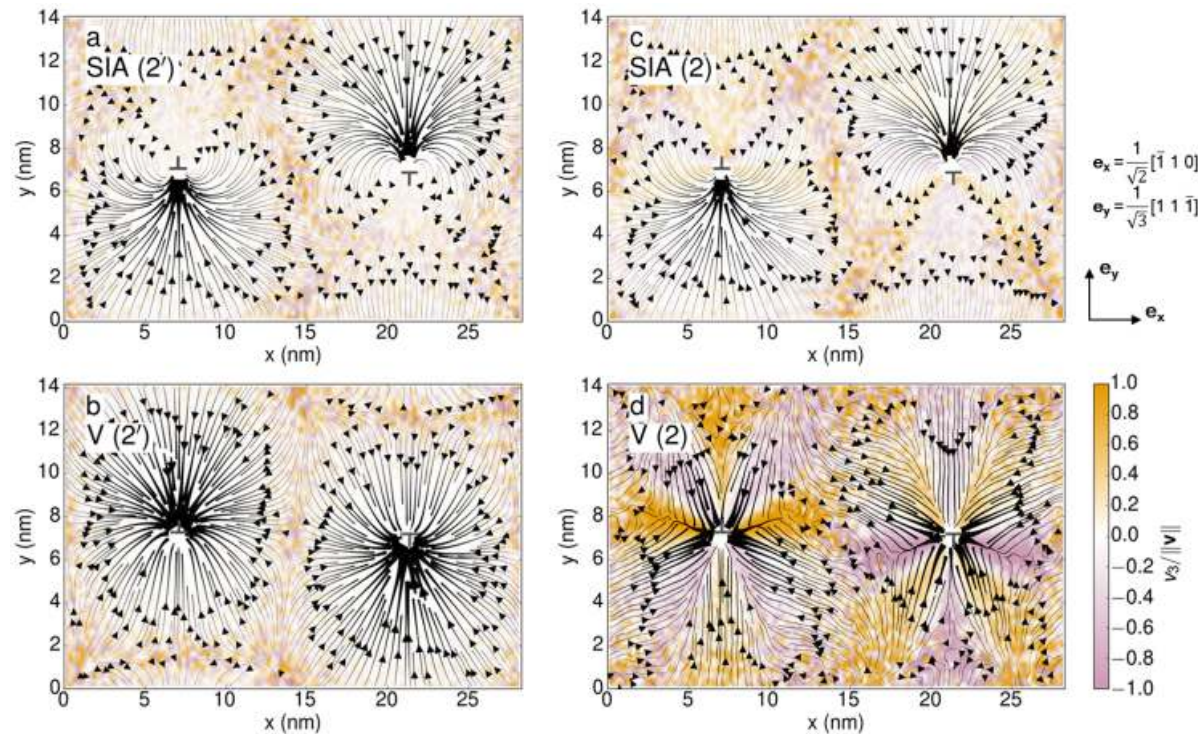

Figure 4: Average fluxes of (a) SIAs and (b) vacancies with isotropic saddle points (case $2^{\prime}$ ), and (c) SIAs and (d) vacancies with real P-tensors (case 2). The flux $\boldsymbol{v}=\left(v_{1}, v_{2}, v_{3}\right)$ is recorded through surfaces between stable positions. The color corresponds to the component of the flux in the $z$-direction $\left(v_{3} /\|\boldsymbol{v}\|\right)$, and the lines represent the flux in the $x y$ plane $\boldsymbol{v}_{x y}=\left(v_{1}, v_{2}\right)$. The width of the lines is here proportional to the norm of the flux $\|\boldsymbol{v}\|=\sqrt{v_{1}^{2}+v_{2}^{2}+v_{3}^{3}}$.

the migration paths of point defects to the dislocations can be extracted from OKMC simulations. The fluxes of point defects are recorded through dividing surfaces between stable atomic positions during OKMC simulations. From these data, average migration paths are obtained. The result is represented in Fig. 4

Results for point defects with isotropic saddle points (case $2^{\prime}$ ) are shown in Fig. 4 a-b. As expected, SIAs migrate and reach the dislocations through their dilated sides and are repelled from the compressed sides. On the contrary, vacancies mostly flow to the compressed sides. The difference is less clear than for interstitials, due to the lower magnitude of the elastic interactions.

When the saddle point anisotropy is taken into account (case 2), we obtain the fluxes in Fig. 4 c-d. These graphs show that the saddle point anisotropy not only has an influence on the sink strength but also on the point defects 
trajectories. Vacancies now migrate to the dislocations to enter through the lateral sides along the Burgers vector direction $\left( \pm \boldsymbol{e}_{x}\right)$. The difference is not so visible for SIAs, as the relative importance of the deviatoric component of their dipole tensors is smaller than for vacancies.

In the case of vacancies, the saddle point anisotropy also gives rise to nonzero component of the flux along the dislocation line direction. Note, however, that by symmetry the flux averaged in the $x y$-plane is zero in this direction. The fluxes along the dislocation line direction can be explained using the diffusion tensor, whose terms can be written 30.

$$
D_{i j}(\boldsymbol{r})=\frac{1}{2} \nu_{0} \sum_{\boldsymbol{h}} h_{i} h_{j} \exp \left(-\frac{E_{\boldsymbol{h}}^{\mathrm{sad}}(\boldsymbol{r})-E^{\mathrm{sta}}(\boldsymbol{r})}{k_{\mathrm{B}} T}\right),
$$

where (see Eqs. (1)-(2))

$$
\begin{aligned}
& E^{\mathrm{sta}}(\boldsymbol{r})=-\sum_{k, l} P_{k l}^{\mathrm{sta}} \epsilon_{k l}(\boldsymbol{r}) \\
& E_{\boldsymbol{h}}^{\mathrm{sad}}(\boldsymbol{r})=E^{\mathrm{m}}-\sum_{k, l} P_{\boldsymbol{h}, k l}^{\mathrm{sad}} \epsilon_{k l}(\boldsymbol{r}+\boldsymbol{h} / 2) .
\end{aligned}
$$

In these equations, $\boldsymbol{h}$ refers to the possible jumps from a stable position $\boldsymbol{r}$ to the neighboring one $\boldsymbol{r}+\boldsymbol{h}$. These possible jumps are represented in Fig. 5 .

In the case of an isotropic saddle point (case $\left.2^{\prime}\right), P_{\boldsymbol{h}, k l}^{\mathrm{sad}}=P_{k l}^{\mathrm{sad}}$. In addition, if we assume that $\epsilon_{k l}(\boldsymbol{r}+\boldsymbol{h} / 2) \approx \epsilon_{k l}(\boldsymbol{r})$, the saddle point energy $E_{\boldsymbol{h}}^{\mathrm{sad}}(\boldsymbol{r})$ no longer depends on the jump and the diffusion tensor becomes

$$
D_{i j}(\boldsymbol{r})=\frac{1}{2} \nu_{0} \exp \left(-\frac{E^{\mathrm{sad}}(\boldsymbol{r})-E^{\mathrm{sta}}(\boldsymbol{r})}{k_{\mathrm{B}} T}\right) \sum_{\boldsymbol{h}} h_{i} h_{j} .
$$

Since

$$
\sum_{\boldsymbol{h}} h_{i} h_{j}=0 \quad \text { if } i \neq j,
$$

an isotropic saddle point leads to a diagonal diffusion tensor. This conclusion also holds for a non uniform strain field, if it is expanded to first order in $\boldsymbol{h}$.

On the contrary, this simplification cannot be made when the saddle point 

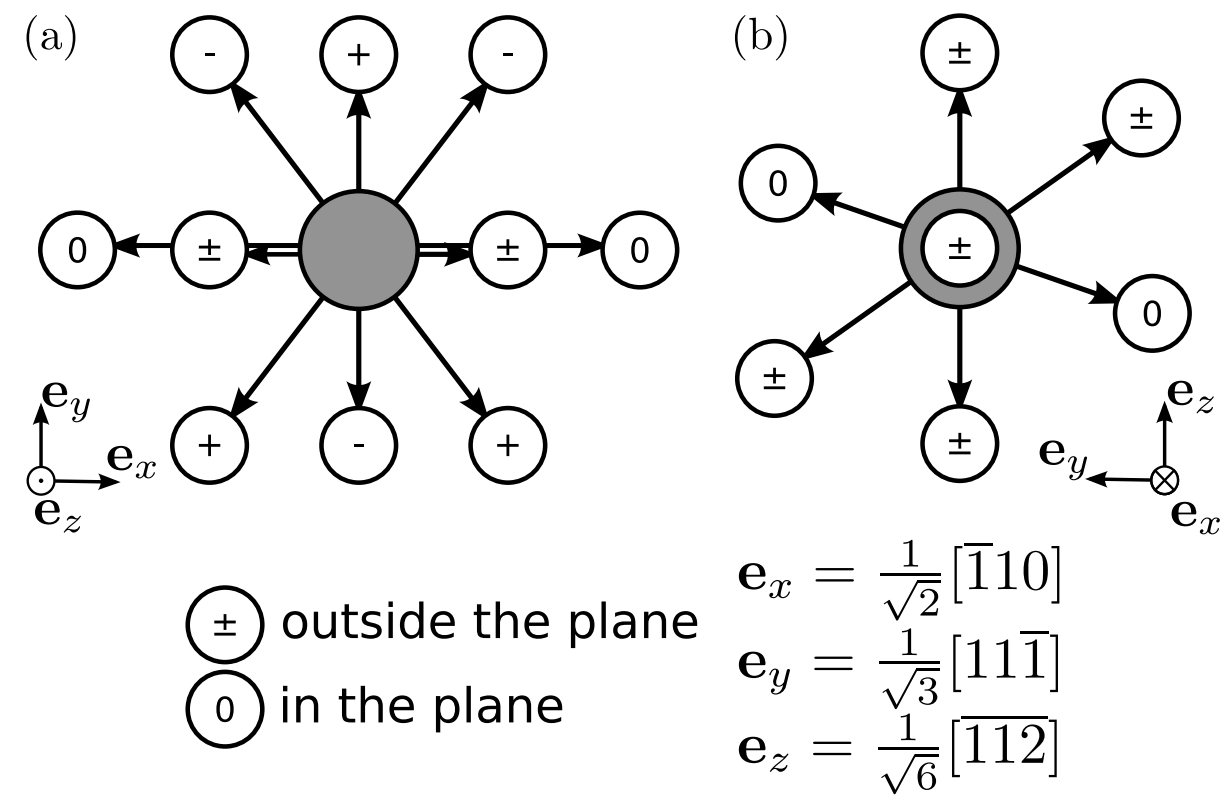

Figure 5: Lattice stable position (gray sphere) and its first nearest neighbor positions (white spheres). Representation (a) in the $x y$ plane and (b) in the $y z$ plane of the simulation box.

dipole tensor is anisotropic. The diffusion tensor terms $D_{i j}$ then depend on

$$
\begin{aligned}
\sum_{\boldsymbol{h}} h_{i} h_{j} \exp \left(-\frac{P_{\boldsymbol{h}, k l}^{\mathrm{sad}} \epsilon_{k l}(\boldsymbol{r}+\boldsymbol{h} / 2)}{k_{\mathrm{B}} T}\right) \approx & \\
& \sum_{\boldsymbol{h}} h_{i} h_{j} \exp \left(-\frac{P_{\boldsymbol{h}, k l}^{\mathrm{sad}} \epsilon_{k l}(\boldsymbol{r})}{k_{\mathrm{B}} T}\right) .
\end{aligned}
$$

Because the strain has a different coupling with each jump $\boldsymbol{h}$, the lattice symmetry is lowered and the different jumps in Fig. 5 no longer cancel each other out. Non-zero off-diagonal terms, in particular $D_{x z}$ and $D_{y z}$, can therefore appear and lead to flux components along the dislocation line direction.

The presence of such a flux component may have some implications on the effect of external stress on dislocation sink strengths. It has been found that the point defect anisotropy at saddle point induces a stress dependence of the dislocation sink strength, known as the "stress-induced preferential absorption due to anisotropic diffusion" (SIPA-AD) [34, 36, 37]. This phenomenon has been shown to be more than one order of magnitude larger than the usual SIPA effect, 
noted SIPA-I, due to the polarisability of point defects [36, 77]. Assessing the relative importance of both mechanisms is crucial in order to describe properly irradiation creep for example. For a uniaxial stress, SIPA-AD manifests itself through a dependency of the sink strength on the direction of the stress with respect to the dislocation line. For the sake of simplicity, let us consider the migration of the vacancy in an $x y$-plane perpendicular to the dislocation line. The saddle point energy reads

$$
E^{\mathrm{sad}}\left(\epsilon^{\mathrm{a}}\right)=E^{\mathrm{sad}}(0)-P_{1}^{\mathrm{sad}} \epsilon_{x^{\prime} x^{\prime}}^{\mathrm{a}}-P_{2}^{\mathrm{sad}} \epsilon_{y^{\prime} y^{\prime}}^{\mathrm{a}}-P_{3}^{\mathrm{sad}} \epsilon_{z z}^{\mathrm{a}},
$$

where $\boldsymbol{\epsilon}^{\mathrm{a}}$ is the applied strain, expressed here in the basis $\left(\boldsymbol{e}_{x^{\prime}}, \boldsymbol{e}_{y^{\prime}}, \boldsymbol{e}_{z}\right)$ where $\boldsymbol{P}^{\mathrm{sad}}$ is diagonal, and $P_{1}^{\mathrm{sad}}=-2.946 \mathrm{eV}, P_{2}^{\mathrm{sad}}=-2.786 \mathrm{eV}$ and $P_{3}^{\mathrm{sad}}=1.000 \mathrm{eV}$ are the three eigenvalues of $\boldsymbol{P}^{\mathrm{sad}}$ (see Eq. (10)). If a tensile stress is applied along the dislocation line $\left(\boldsymbol{e}_{z}\right)$, the energy becomes

$$
E^{\mathrm{sad}}\left(\boldsymbol{\epsilon}^{\mathrm{a}}\right)=E^{\mathrm{sad}}(0)-2.946 \nu \epsilon^{\mathrm{a}}-2.786 \nu \epsilon^{\mathrm{a}}-1.000 \epsilon^{\mathrm{a}} .
$$

If the stress is in the plane, for example along $\boldsymbol{e}_{x^{\prime}}$, it reads

$$
E^{\mathrm{sad}}\left(\epsilon^{\mathrm{a}}\right)=E^{\mathrm{sad}}(0)+2.946 \nu \epsilon^{\mathrm{a}}-2.786 \nu \epsilon^{\mathrm{a}}+1.000 \epsilon^{\mathrm{a}} .
$$

Therefore it is easily seen that providing vacancies migrate in a plane normal to the dislocation line, a tensile stress along the line will decrease the migration barrier and thus increase the sink strength, whereas a tensile stress in the jump direction will have the opposite effect. If the vacancy does not migrate in this plane, the effect can be lower. In the works dealing with SIPA-AD, the large magnitude of the SIPA-AD effect has been obtained by assuming that point defect fluxes are perpendicular to the dislocation line [36, 77]. However, we have seen that when a three-dimensional model is used, saddle point anisotropy leads to fluxes with components along the dislocation line. Therefore, it is not clear whether the magnitude of the SIPA-AD will remain as large compared to SIPA-I. Additional work is needed to evaluate precisely the importance of both mechanisms, by studying specifically different stress levels and directions [78]. 


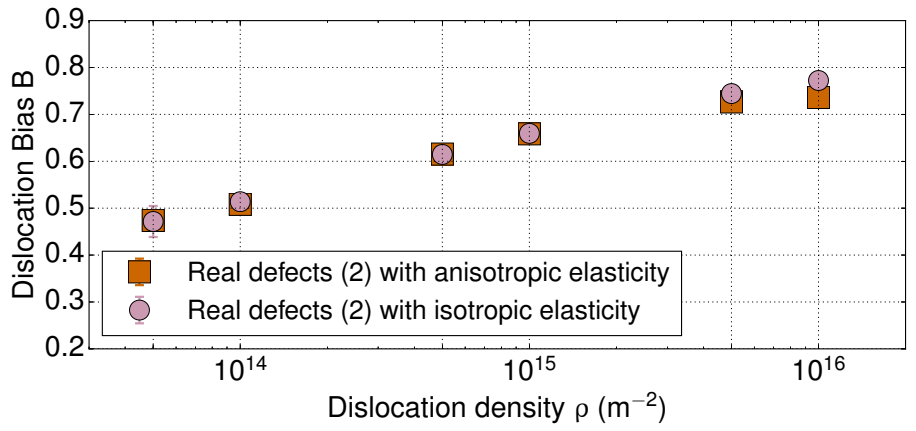

Figure 6: Dislocation bias obtained for real defects (case 2) with interpolation of the analytical solution in Fourier space for the strain, with isotropic and anisotropic elasticity.

\subsection{Importance of elastic moduli tensor anisotropy}

Point defect anisotropy at saddle point has been identified as one of the main parameters influencing the sink strength value in isotropic elasticity. Although aluminum is a weakly anisotropic material ( $A=1.21$, see Tab. 1), it can be worth investigating the effect of the anisotropy of the elastic moduli tensor on the sink strength.

The results obtained for the dislocation bias are presented in Fig. 6. In this figure, both results are obtained with calculations using the analytical solution in Fourier space for the strain field 48, 49, 50. With anisotropic elasticity, only a small variation of the bias values can be observed. It is much less important than the variation induced by the saddle point anisotropy. The influence of the elastic moduli tensor anisotropy is therefore far less important than the one of the point defect anisotropy. Consequently, as far as aluminum is concerned, the calculations can be done with isotropic elasticity. A similar conclusion was drawn in the case of iron, for which it was seen that the change in the sink strength is lower than $10 \%$ when elastic constants are changed from their values at $0 \mathrm{~K}(A=2.3)$ to the ones at $1000 \mathrm{~K}(A=4.5)[79$. 


\section{Spherical cavity}

\subsection{Case definition}

To compute the cavity sink strengths and bias, the cubic simulation box of size $d$ contains a single centered spherical cavity, as illustrated in Fig. 7. This cavity is a perfect sink with a constant radius $r_{c}$, with a capture radius of $d_{\text {reac }}=r_{c}+r_{\mathrm{PD}}$ where $r_{\mathrm{PD}}$ is the point defect radius $\left(r_{\mathrm{PD}}=0.16 \mathrm{~nm}\right)$. The cavity density only depends on the box dimensions and is given by $\rho=1 / d^{3}$, so different densities can be studied by varying the box dimension $d$.

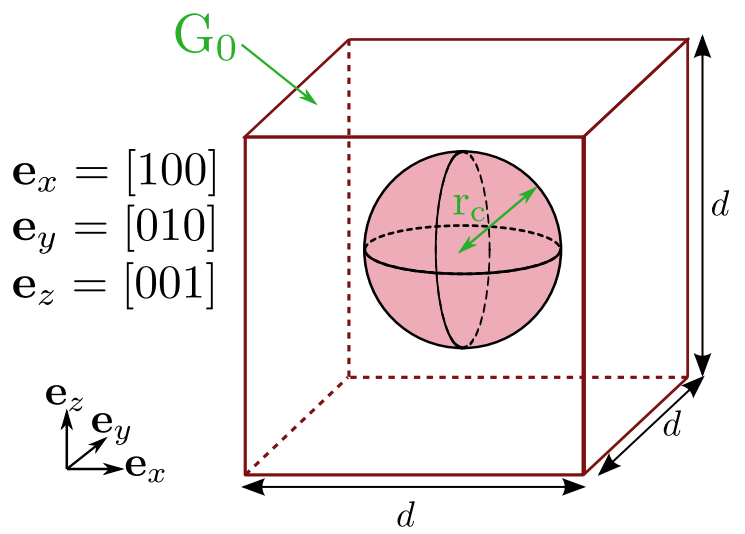

Figure 7: Configuration of the simulation box containing a spherical cavity.

The strain field generated by a cavity is calculated after the expressions given in [80, 39]:

$$
\epsilon_{i j}(\boldsymbol{r})=\frac{\left(-\frac{2 \gamma}{r_{c}}\right)}{4 \mu}\left(\frac{r_{c}}{r}\right)^{3}\left(\delta_{i j}-\frac{3 x_{i} x_{j}}{r^{2}}\right) .
$$

In this equation, $\mu$ is the shear modulus of the material, the coordinates in the box $x, y, z$ are noted $x_{1}, x_{2}, x_{3}$ for convenience, with $r=\sqrt{x_{1}^{2}+x_{2}^{2}+x_{3}^{2}}$, and $\gamma$ is the surface tension, set to the typical value of $1 \mathrm{~J}^{-\mathrm{m}^{-2}}$ [39].

From Eq. 25, it can be seen that $\operatorname{Tr}(\boldsymbol{\epsilon})=0$. As a consequence, for defects having an isotropic dipole tensor $\boldsymbol{P}=P_{0} \boldsymbol{I}$, the energy induced by the elastic field is $E=-P_{0} \operatorname{Tr}(\boldsymbol{\epsilon})=0$. Therefore when both vacancies and SIAs have isotropic dipole tensors, e.g. in the usual approximation of case 1, the bias is zero, and the cavity is a neutral sink. 
The simulations involving defects with isotropic dipole tensors at both stable and saddle points (case 1 and case $2^{\prime \prime}$ ) are then equivalent to case 0 , with no interactions. Consequently, only the cases of non interacting defects (case 0 ), real defects (case 2) and defects simplified at saddle points (case $2^{\prime}$ ) are considered here.

\subsection{Sink strength and bias}
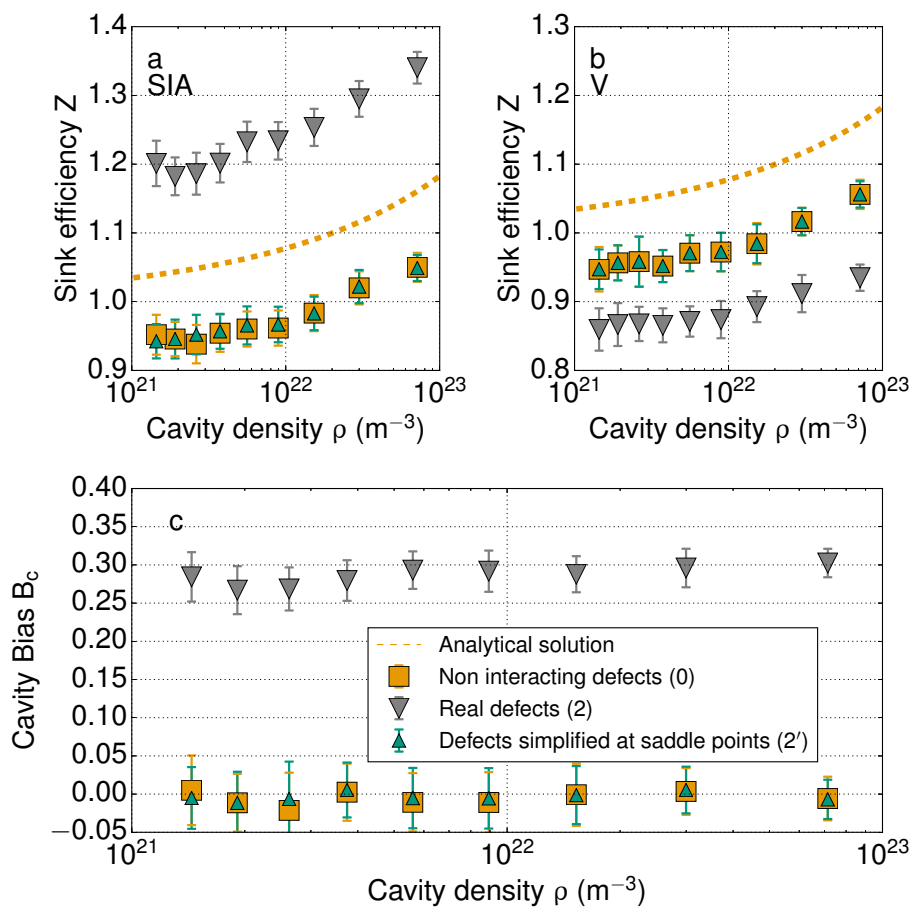

Figure 8: Cavity sink efficiency for (a) SIAs and (b) vacancies, (c) bias, for a cavity of radius $1 \mathrm{~nm}$.

The cavity sink strength and bias are calculated for a cavity of radius $r_{c}=$ $1 \mathrm{~nm}$, for a density between $10^{21}$ and $10^{23}$ cavities per $\mathrm{m}^{3}$, corresponding to densities commonly measured in irradiated materials [67]. The sink efficiencies $Z$ are then calculated by

$$
Z=\frac{k^{2}}{4 \pi d_{\mathrm{reac}} \rho} .
$$


Results are presented in Fig. 8

As in the case of dislocations, a good agreement is obtained between the sink strengths for non interacting defects (case 0, yellow squares) and the analytical expression for the cavity sink strength 68, validating our approach. The small difference can be explained by the boundary conditions at the cavity surface, in the same way as for the dislocation.

It is particularly interesting to notice how the elastic interactions influence the sink strength values. The results obtained for real defects (case 2, gray downpointing triangles) show that the interactions increase the cavity sink strength for SIAs, but slightly reduce the sink strength for vacancies as compared to the case without elastic interactions. This yields a positive bias of 0.3 in average for the densities studied. The bias weakly depends on the cavity density, because of the short range of interactions. This bias value shows that the cavity cannot be considered as a neutral sink when the elastic interactions are taken into account.

The sink strengths obtained for point defects with isotropic saddle points (case $2^{\prime}$, green triangles) are equal to the ones obtained for non interacting defects, so the bias is zero. Therefore, results show that the saddle point anisotropy of point defects alone is responsible for the increase of the bias value. The stable point anisotropy does not influence the sink strength nor the bias, which is consistent with the results obtained for the dislocation.

Fig. 9 presents the cavity bias for a density of $3.7410^{21} \mathrm{~m}^{-3}$, for cavity radii from $1 \mathrm{~nm}$ to $5 \mathrm{~nm}$, with consideration of point defects anisotropy (case 2). The cavity bias decreases when the cavity radius increases due to the decrease in $1 / r_{\mathrm{c}}$ in Eq. 25, but even for a radius of $5 \mathrm{~nm}$ the bias is still above 0.1 , which indicates that the cavity is a biased sink.

\subsection{Analysis and discussion}

In the same way as for dislocations, cavity sink strengths have been determined by analytical calculations, taking into account the diffusion anisotropy [39]. With different boundary conditions as those used in our simulations, and under the assumptions that the cavity is small with respect to the simulation cell and 


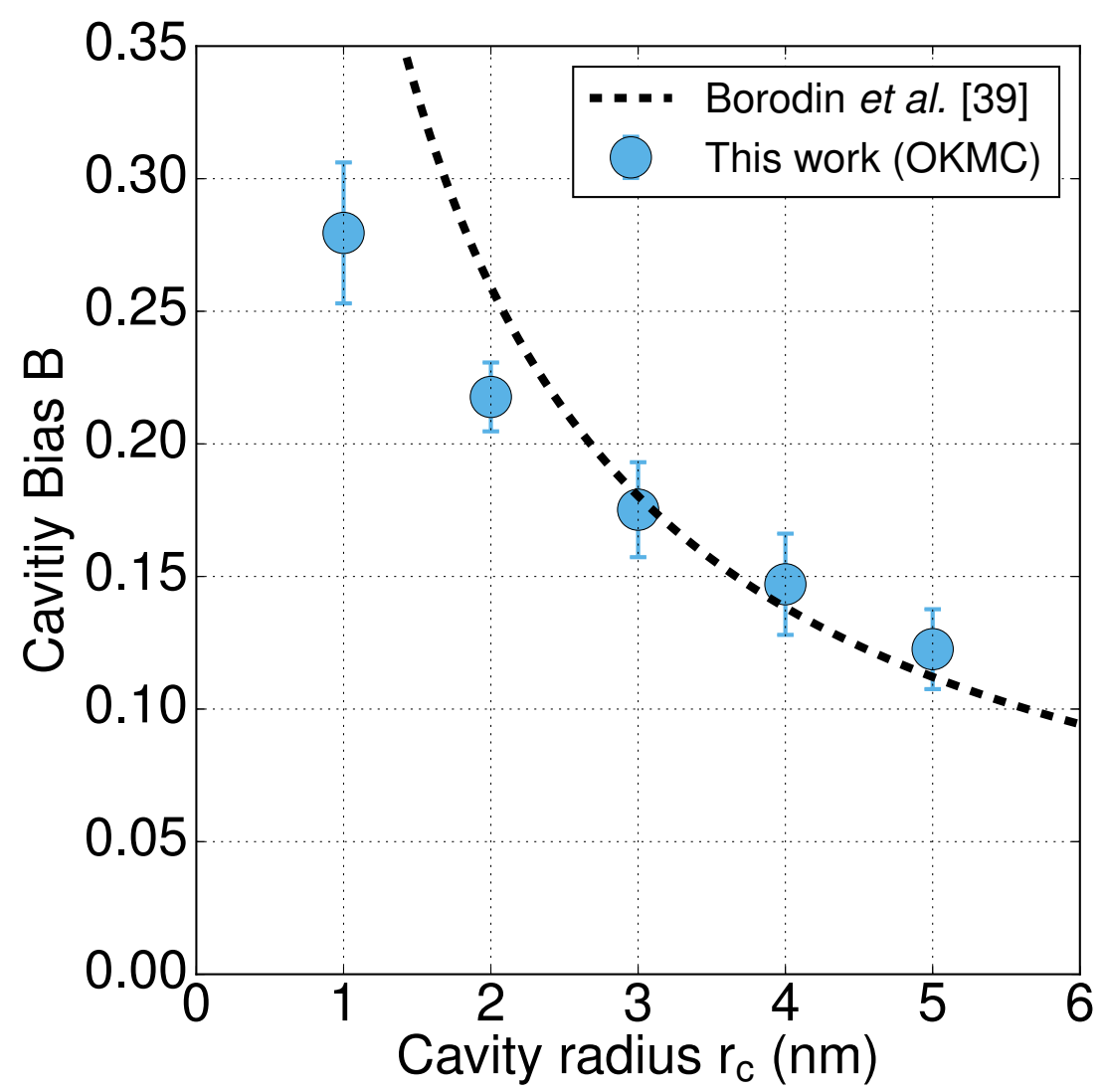

Figure 9: Cavity bias $B$ as a function of the cavity radius $r_{c}$, for real defects (case 2), and the analytical expression computed from [39]. The cavity density is $3.7410^{21} \mathrm{~m}^{-3}$.

that the strain is low, the expression obtained for the sink strength is remarkably simple: it depends on the components of the dipole tensor and varies as $1 / r_{\mathrm{c}}$. The bias values obtained from these expressions are represented in Fig. 9 This analytical expression is in agreement with our simulation results for the larger cavities, but the values differ for the small radii, for which the strain field is stronger. For our simulation parameters, these calculations show that for a cavity of radius $1 \mathrm{~nm}$, the sink strength for vacancies is reduced by a factor 0.69 when the anisotropy at saddle point is taken into account, while the one for 
SIAs increases by a factor 1.29. In our simulations, the reduction for vacancies is less marked, whereas the value for SIAs is very close. Therefore, the bias value is higher in the analytical approach $(B=0.46)$.

More in-depth investigation of the effect of saddle point anisotropy can be obtained by extracting point defect trajectories. They are represented in Fig. 10 in the $x y$-plane, for both isotropic (case $2^{\prime}, \mathrm{a}-\mathrm{b}$ ) and anisotropic (case 2, cd) saddle points. In the case of isotropic saddle point, the average fluxes of both SIAs and vacancies are normal to the cavity. This corresponds to the fluxes that would be obtained without interactions between point defects and the sink. When the saddle point is anisotropic, trajectories of SIAs are not markedly changed. On the contrary, trajectories of vacancies are curved close to the cavity.

Fig. 11 presents the probability of absorption on the surface of the cavity for a radius of $1 \mathrm{~nm}$. These results confirm that the probability of absorption of SIAs is almost homogeneous on the cavity surface, with a small increase along the $\langle 111\rangle$ directions. On the contrary, Fig. 11 b shows that the probability of absorption of vacancies is strongly anisotropic. Vacancies are absorbed almost only through the $\langle 100\rangle$ directions. In a similar way, anisotropic concentration fields around the cavity were found by Borodin et al. 39. Qualitatively, it can be explained by the curvature of the point defect trajectories (Fig. 10-d), which converge to the $\langle 100\rangle$ directions. In general, as emphasized in the case of the dislocation, vacancies tend to migrate more easily if the strain along the jump direction is negative (such defects are called "F-type defects" by Woo 38]). Tangential strain due to the cavity is negative, while normal strain is positive. Therefore, defects tend to migrate tangentially close to the cavity, which reduces the point defect absorption and the sink strength. This effect is more or less strong, depending on the orientation of jumps with respect to the strain field of the cavity. This lattice effect is a signature of the saddle point anisotropy. In order to more precisely assess this trajectory curvature effect very near to the cavity, more involved simulations, including other energy terms that may be important at low distance [45, should be performed. 

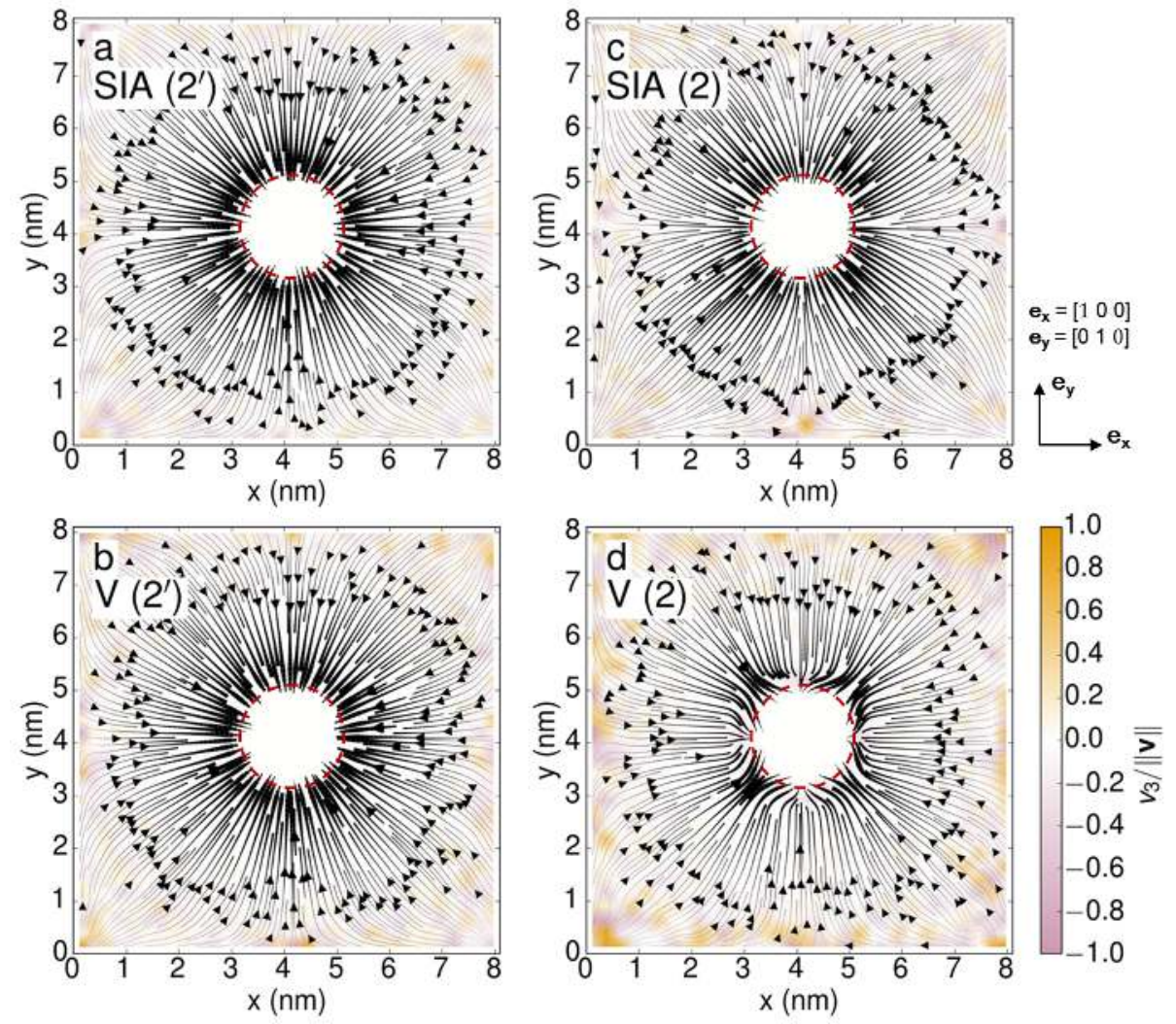

Figure 10: Average fluxes of (a) SIAs and (b) vacancies with isotropic saddle points (case $2^{\prime}$ ), and (c) SIAs and (d) vacancies with real $\mathbf{P}$-tensors (case 2). The same convention is applied as in Fig. 4

\section{Conclusion}

In this work, OKMC simulations have been performed to evaluate sink strengths and bias values of a straight dislocation and of a spherical cavity in aluminum. The elastic interactions between the sink and the migrating point defects are explicitly modeled and the point defects are represented by their elastic dipole tensors (P-tensors), which are computed by DFT calculations. The influence of the saddle point anisotropy on the sink strengths and bias has been quantitatively assessed.

Results show that the values of sink strengths and bias are strongly increased 


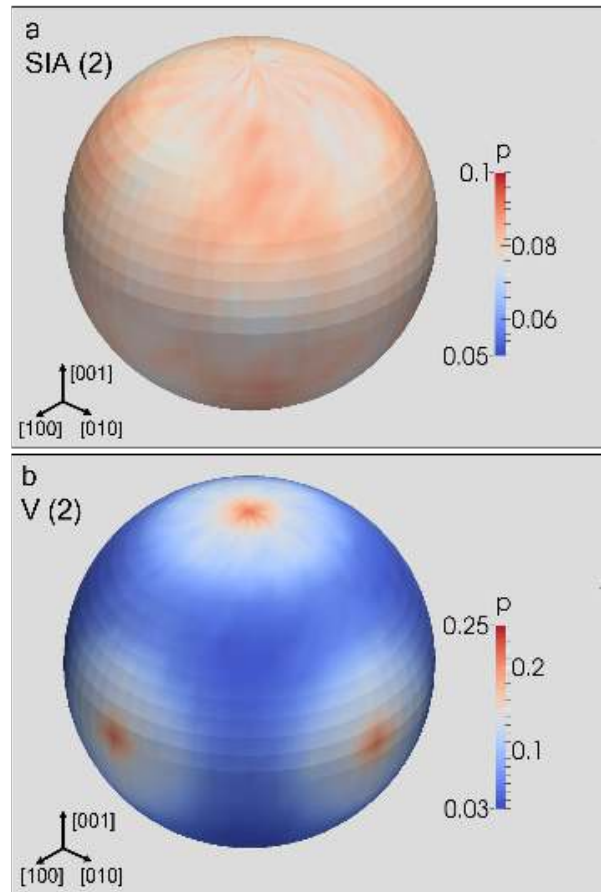

Figure 11: Probability density of absorption $p$ of (a) SIAs and (b) vacancies (case 2) on the surface of the cavity, for a cavity radius of $1 \mathrm{~nm}$.

by the elastic interactions, for both the dislocation and the cavity. The study of the different approximations of the $\mathbf{P}$-tensors highlights the influence of the saddle point anisotropy of defects. It leads to the cavity being a biased sink with a bias of almost 0.3 for a radius of $1 \mathrm{~nm}$, which reduces as the radius increases.

The influence of the saddle point anisotropy has been enlightened by the study of the point defects migration paths. We have shown that the saddle point anisotropy leads to more complex trajectories than in the isotropic case, especially for vacancies which have a more anisotropic saddle point than interstitials. Near the dislocation, vacancies migrate along the Burgers vector direction, and not only through the compressed side. The vacancy flux has a non-zero component along the dislocation line direction. Near a cavity, vacancies tend to migrate tangentially and be absorbed through the $\langle 100\rangle$ directions, while the absorption of SIAs is nearly isotropic. 
Similar results are expected for other FCC metals, as dipole tensors have the same features [29. These results have implications on swelling and irradiation creep rates, and also suggest that the saddle point anisotropy of vacancies could play a significant role in many FCC based materials, in particular when solidification, heat treatments and/or deformation processes have led to a supersaturation of vacancies. In addition, this anisotropy could be of importance in coherent multi-phase materials, such as nickel based superalloys, in which vacancies diffuse in an inhomogeneous strain field resulting from both the internal multiphase microstructure and the thermo-mechanical treatment.

\section{Appendix A. Comparison of OKMC results and analytical calcula- tions for straight dislocations (case 1)}

In this section we compare the OKMC results for case 1 (spherical inclusions), for straight dislocations, with the analytical calculation provided by Rauh and Simon [74. This analytical study corresponds to a single dislocation with a prescribed concentration at a distance $R$ from the dislocation position. The effect of the strain field of other dislocations is therefore not taken into account.

In order to explain the discrepancy observed in Fig. 3, the drift-diffusion equation has also been solved on two different cases (Fig. A.12), using the finite element (FE) method. More details about the methodology can be found in Ref. 23. In the first case (Fig. A.12-(a)), the concentration is imposed at the outer boundary of the computation domain, corresponding to the analytical treatment from [74]. The sink strength is deduced from the flux to the dislocation. In the second case (Fig. A.12 (b)), a source term is imposed, corresponding to our OKMC simulations. The sink strength is deduced from the average concentration of defects, using Eq. (5). In both cases, the effect of the strain field of surrounding dislocations is taken into account.

Results are compared in Fig. A.13. A very good agreement is obtained between FE simulations with imposed creation rate and OKMC simulations. On the contrary, FE simulations with imposed concentration tends to the an- 
alytical solution for low dislocation densities, whereas they depart from each other as the dislocation density increases. This means that the discrepancy between the analytical solution and the OKMC results mostly comes from the different boundary conditions, whereas at high dislocation densities the effect of surrounding dislocation strain fields also plays a role in the difference.

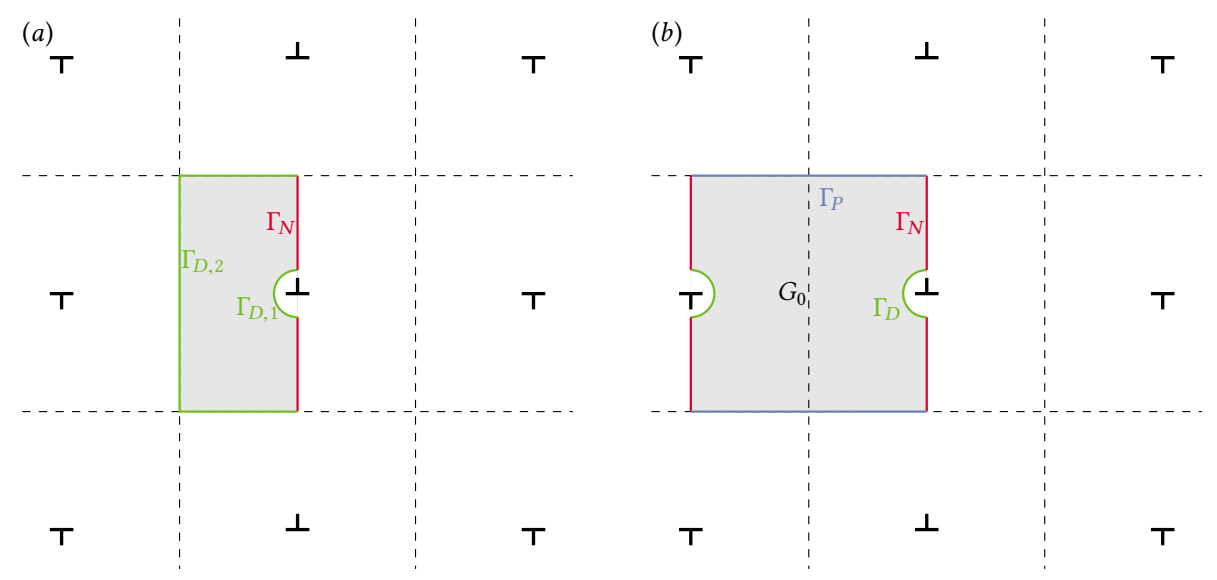

Figure A.12: Computation domains for FE calculations (in grey). (a) Concentration is imposed on the outer boundary $\left(\Gamma_{D, 2}\right)$. Concentration is zero on $\Gamma_{D, 1}$ and the normal flux is zero on $\Gamma_{N}$. (b) Creation rate $G_{0}$ is imposed in the volume. Concentration is zero on $\Gamma_{D}$, the normal flux is zero on $\Gamma_{N}$ and periodic boundary conditions are used on $\Gamma_{P}$.

[1] S. H. Goods, L. M. Brown, The nucleation of cavities by plastic deformation, Acta Metall. 27 (1979) 1.

[2] V. G. Gavriljuk, V. N. Bugaev, Y. N. Petrov, A. V. Tarasenko, Hydrogeninduced equilibrium vacancies in fcc iron-base alloys, Scr. Mater. 34 (1996) 903.

[3] T. Neeraj, R. Srinivasan, J. Li, Hydrogen embrittlement of ferritic steels: Observations on deformation microstructure, nanoscale dimples and failure by nanovoiding, Acta Mater. 60 (2012) 5160.

[4] J. Takamura, Quenched-in vacancies and quenching strains in gold, Acta Metall. 9 (1961) 547. 


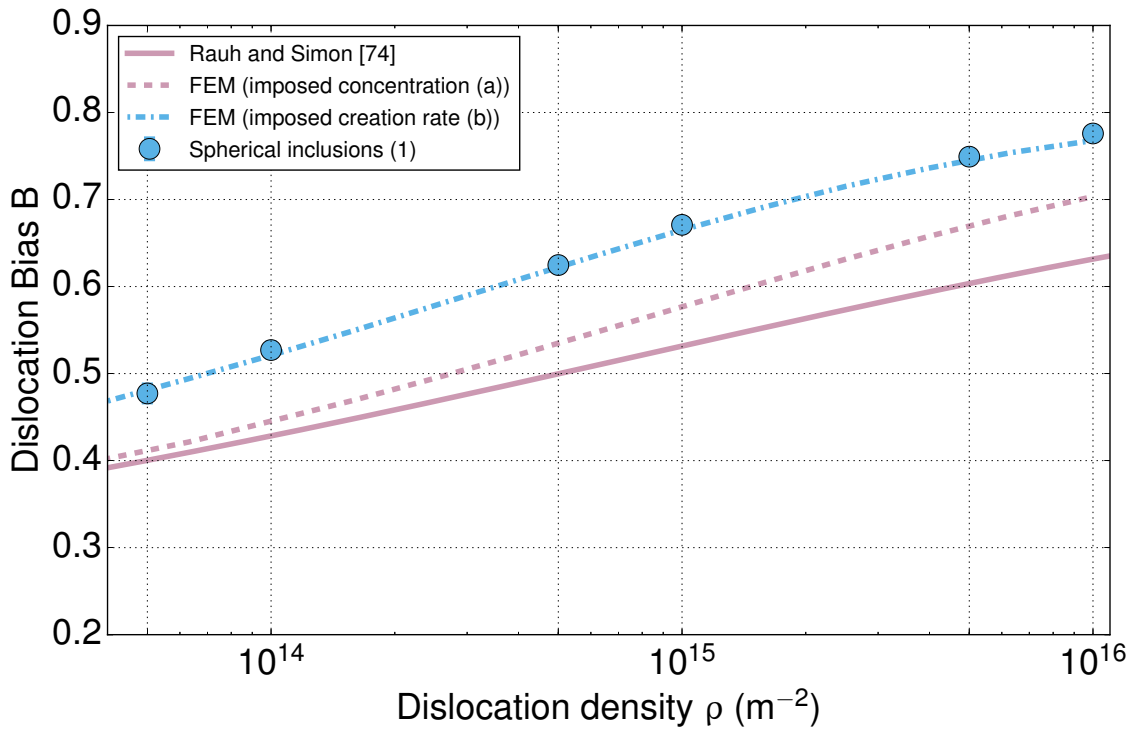

Figure A.13: Bias of straight dislocations when point defects are considered as spherical inclusions (case 1), obtained with various approaches: analytical calculation by Rauh and Simon [74, FE calculations with two different boundary conditions and OKMC calculations. 
[5] P. B. Hirsch, J. Silcox, R. E. Smallman, K. H. Westmacott, Dislocation loops in quenched aluminum, Philos. Mag. 3 (1958) 897.

[6] G. Thomas, R. H. Willens, Defects in aluminum quenched from the liquid state, Acta Metall. 12 (1964) 191.

[7] G. W. Greenwood, A. J. E. Foreman, D. E. Rimmer, The role of vacancies and dislocations in the nucleation and growth of gas bubbles in irradiatied fissile materials, J. Nucl. Mater. 4 (1959) 305.

[8] P. T. Heald, M. V. Speight, Steady-state irradiation creep, Philos. Mag. 29 (1974) 1075.

[9] L. K. Mansur, Irradiation creep by climb-enabled glide of dislocations resulting from preferred absorption of point defects, Philos. Mag. A 39 (1979) 497.

[10] A. D. Brailsford, R. Bullough, The rate theory of swelling due to void growth in irradiated metals, J. Nucl. Mater. 44 (1972) 121.

[11] S. I. Golubov, A. M. Ovcharenko, A. V. Barashev, B. N. Singh, Grouping method for the approximate solution of a kinetic equation describing the evolution of point-defect clusters, Philos. Mag. A 81 (2001) 643.

[12] A. Hardouin-Duparc, C. Moingeon, N. Smetniansky-de-Grande, A. Barbu, Microstructure modelling of ferritic alloys under high flux $1 \mathrm{MeV}$ electron irradiations, J. Nucl. Mater. 302 (2002) 143.

[13] D. Xu, B. D. Wirth, M. Li, M. A. Kirk, Combining in situ transmission electron microscopy irradiation experiments with cluster dynamics modeling to study nanoscale defect agglomeration in structural materials, Acta Mater. 60 (2012) 4286.

[14] T. Jourdan, G. Bencteux, G. Adjanor, Efficient simulation of kinetics of radiation induced defects: A cluster dynamics approach, J. Nucl. Mater. 444 (2014) 298. 
[15] M. J. Makin, G. P. Walters, A. J. E. Foreman, The void swelling behaviour of electron irradiated type 316 austenitic steel, J. Nucl. Mater. 95 (1980) 155.

[16] G. P. Walters, The electron irradiation of pure Fe-Cr-Ni alloys in temperature range 400 to $700^{\circ} \mathrm{C}$, J. Nucl. Mater. 136 (1985) 263.

[17] S. I. Golubov, B. N. Singh, H. Trinkaus, On recoil-energy-dependent defect accumulation in pure copper Part II. Theoretical treatment., Philos. Mag. A 81 (2001) 2533.

[18] T. Okita, W. G. Wolfer, A critical test of the classical rate theory for void swelling, J. Nucl. Mater. 327 (2004) 130.

[19] Z. Chang, P. Olsson, D. Terentyev, N. Sandberg, Dislocation bias factors in fcc copper derived from atomistic calculations, J. Nucl. Mater. 441 (2013) 357.

[20] C. H. Woo, W. S. Liu, M. S. Wuschke, A Finite-difference Calculation of Point Defect Migration into a Dislocation Loop, Technical Report AECL6441, Atomic Energy of Canada Limited, 1979.

[21] R. Bullough, D. W. Wells, J. R. Willis, M. H. Wood, The interaction energy between interstitial atoms and dislocations and its relevance to irradiation damage processes, in: M. F. Ashby, R. Bullough, C. S. Hartley, J. P. Hirth (Eds.), Dislocation Modelling of Physical Systems, Pergamon, 1981, p. 116.

[22] V. I. Dubinko, A. S. Abyzov, A. A. Turkin, Numerical evaluation of the dislocation loop bias, J. Nucl. Mater. 336 (2005) 11.

[23] T. Jourdan, Influence of dislocation and dislocation loop biases on microstructures simulated by rate equation cluster dynamics, J. Nucl. Mater. 467 (2015) 286.

[24] H. Rouchette, L. Thuinet, A. Legris, A. Ambard, C. Domain, Quantitative phase field model for dislocation sink strength calculations, Comp. Mater. Sci. 88 (2014) 50. 
[25] H. L. Heinisch, B. N. Singh, S. I. Golubov, The effects of one-dimensional glide on the reaction kinetics of interstitial clusters, J. Nucl. Mater. 283 (2000) 737.

[26] L. Malerba, C. S. Becquart, C. Domain, Object kinetic Monte Carlo study of sink strengths, J. Nucl. Mater. 360 (2007) 159.

[27] V. Jansson, L. Malerba, A. De Backer, C. S. Becquart, C. Domain, Sink strength calculations of dislocations and loops using OKMC, J. Nucl. Mater. 442 (2013) 218.

[28] A. B. Sivak, V. M. Chernov, V. A. Romanov, P. A. Sivak, Kinetic MonteCarlo simulation of self-point defect diffusion in dislocation elastic fields in bcc iron and vanadium, J. Nucl. Mater. 417 (2011) 1067.

[29] A. Vattré, T. Jourdan, H. Ding, M.-C. Marinica, M. J. Demkowicz, Nonrandom walk diffusion enhances the sink strength of semicoherent interfaces, Nat. Commun. 7 (2016) 10424.

[30] P. H. Dederichs, K. Schroeder, Anisotropic diffusion in stress fields, Phys. Rev. B 17 (1978) 2524.

[31] J. S. Koehler, Diffusion of lattice defects in a stress field, Phys. Rev. 181 (1969) 1015.

[32] H. K. Birnbaum, B. L. Eyre, W. Drotning, The effect of diffusivity gradients on diffusion to dislocations, Philos. Mag. 23 (1971) 847.

[33] K. Schroeder, K. Dettmann, Diffusion reactions in long range potentials, Z. Phys. B 22 (1975) 343.

[34] C. N. Tomé, H. A. Cecatto, E. J. Savino, Point-defect diffusion in a strained crystal, Phys. Rev. B 25 (1982) 7428.

[35] I.-W. Chen, Anisotropic diffusion of point defects to edge dislocations, J. Nucl. Mater. 125 (1984) 52. 
[36] B. C. Skinner, C. H. Woo, Shape effect in the drift diffusion of point defects into straight dislocations, Phys. Rev. B 30 (1984) 3084.

[37] V. A. Borodin, A. I. Ryazanov, The effect of diffusion anisotropy on dislocation bias and irradiation creep in cubic lattice materials, J. Nucl. Mater. 210 (1994) 258.

[38] C. H. Woo, Intrinsic bias differential between vacancy loops and interstitial loops, J. Nucl. Mater. 107 (1982) 20.

[39] V. A. Borodin, A. I. Ryazanov, C. Abromeit, Void bias factors due to the anisotropy of the point defect diffusion, J. Nucl. Mater. 207 (1993) 242.

[40] G. Subramanian, D. Perez, B. P. Uberuaga, C. N. Tomé, A. F. Voter, Method to account for arbitrary strains in kinetic Monte Carlo simulations, Phys. Rev. B 87 (2013) 144107.

[41] D. T. Gillespie, A general method for numerically simulating the stochastic time evolution of coupled and chemical reactions, J. Comput. Phys. 2 (1976) 403.

[42] A. B. Bortz, M. H. Kalos, J. L. Lebowitz, A new algorithm for Monte Carlo simulation of Ising spin systems, J. Comput. Phys. 17 (1975) 10.

[43] E. Kröner, Die Versetzung als elementare Eigenspannungsquelle, Z. Naturforsch., A: Phys. Sci. 11 (1956) 969.

[44] R. Siems, Mechanical interactions of point defects, Phys. Stat. Sol. 30 (1968) 645.

[45] W. G. Wolfer, Segregation of point defects by internal stress fields, in: M. T. Robinson, F. W. Young, Jr. (Eds.), Fundamental Aspects of Radiation Damage in Metals, volume II, p. 812.

[46] A. I. Ryazanov, D. G. Sherstennikov, Void bias factor in materials with weak cubic anisotropy, J. Nucl. Mater. 186 (1991) 33. 
[47] J. L. Tallon, A. Wolfenden, Temperature dependence of the elastic constants of aluminum, J. Phys. Chem. Solids 40 (1979) 831.

[48] A. Finel, D. Rodney, Phase field methods and dislocations, in: MRS Fall Meeting Y4.9, Boston, 2000, p. 652.

[49] S. Y. Hu, L. Q. Chen, Solute segregation and coherent nucleation and growth near a dislocation - a phase-field model integrating defect and phase microstructures, Acta Mater. 49 (2001) 463.

[50] D. Rodney, Y. Le Bouar, A. Finel, Phase field methods and dislocations, Acta Mater. 51 (2003) 17.

[51] J. P. Hirth, J. Lothe, Theory of Dislocations, Wiley-lnterscience, 1982.

[52] P. T. Heald, M. V. Speight, Point defect behaviour in irradiated materials, Acta Metall. 23 (1975) 1389.

[53] H. Flyvbjerg, H. G. Petersen, Error estimates on averages of correlated data, J. Chem. Phys. 91 (1989) 461.

[54] G. Kresse, J. Hafner, Ab initio molecular dynamics for liquid metals, Phys. Rev. B 47 (1993) 558.

[55] G. Kresse, J. Hafner, Ab initio molecular-dynamics simulation of the liquidmetal amorphous-semiconductor transition in germanium, Phys. Rev. B 49 (1994) 14251.

[56] G. Kresse, J. Furthmüller, Efficiency of ab-initio total energy calculations for metals and semiconductors using a plane-wave basis set, Comp. Mater. Sci. 6 (1996) 15.

[57] G. Kresse, J. Furthmüller, Efficient iterative schemes for ab initio totalenergy calculations using a plane-wave basis set, Phys. Rev. B 54 (1996) 11169. 
[58] J. P. Perdew, K. Burke, M. Ernzerhof, Generalized gradient approximation made simple, Phys. Rev. Lett. 77 (1996) 3865.

[59] G. Henkelman, B. P. Uberuaga, H. Jnsson, A climbing image nudged elastic band method for finding saddle points and minimum energy paths, J. Chem. Phys. 113 (2000) 9901.

[60] V. Spirić, L. E. Rehn, K.-H. Robrock, W. Schilling, Anelastic relaxation due to single self-interstitial atoms in electron-irradiated Al, Phys. Rev. B 15 (1977) 672.

[61] W. Schilling, Self-interstitial atoms in metals, J. Nucl. Mater. 69 \& 70 (1978) 465.

[62] W. G. Wolfer, 1.01 - Fundamental properties of defects in metals, in: R. J. Konings (Ed.), Comprehensive Nuclear Materials, Elsevier, Oxford, 2012, p. 1.

[63] P. T. Heald, The preferential trapping of interstitials at dislocations, Philos. Mag. 31 (1975) 551.

[64] C. Jiang, N. Swaminathan, J. Deng, D. Morgan, I. Szlufarska, Effect of grain boundary stresses on sink strength, Mater. Res. Lett. 2 (2014) 100.

[65] W. G. Wolfer, The dislocation bias, J. Comput. Aided Mater. Des. 14 (2007) 403.

[66] W. P. Kuykendall, W. Cai, Conditional convergence in two-dimensional dislocation dynamics, Model. Simul. Mater. 21 (2013) 055003.

[67] H. R. Brager, J. L. Straalsund, Defect development in neutron irradiated type 312 stainless steel, J. Nucl. Mater. 46 (1973) 134.

[68] F. A. Nichols, On the estimation of sink-absorption terms in reaction-ratetheory analysis of radiation damage, J. Nucl. Mater. 75 (1978) 32. 
[69] J. Hou, X.-S. Kong, X.-Y. Li, X. Wu, C. S. Liu, J.-L. Chen, G.-N. Luo, Modification on theory of sink strength: An Object Kinetic Monte Carlo study, Comp. Mater. Sci. 123 (2016) 148.

[70] H. Rouchette, Sink efficiency calculation of dislocations in irradiated materials by phase-field modelling, Ph.D. thesis, Université de Lille 1, 2015.

[71] F. S. Ham, Stress-Assisted Precipitation on Dislocations, J. Appl. Phys. $30(1959) 915$.

[72] I. G. Margvelashvili, Z. K. Saralidze, Influence of an elastic field of a dislocation on steady-state diffusion fluxes of point defects, Sov. Phys. Solid State 15 (1974) 1774.

[73] W. G. Wolfer, M. Ashkin, Diffusion of vacancies and interstitials to edge dislocations, J. Appl. Phys. 47 (1976) 791.

[74] H. Rauh, D. Simon, On the Diffusion Process of Point Defects in the Stress Field of Edge Dislocations, Phys. Status Solidi A 46 (1978) 499.

[75] A. B. Sivak, P. A. Sivak, Efficiency of dislocations as sinks of radiation defects in fcc copper crystal, Crystallogr. Rep. 59 (2014) 407.

[76] H. Rouchette, L. Thuinet, A. Legris, A. Ambard, C. Domain, Influence of shape anisotropy of self-interstitials on dislocation sink efficiencies in $\mathrm{Zr}$ : Multiscale modeling, Phys. Rev. B 90 (2014) 014104.

[77] C. H. Woo, Irradiation creep due to elastodiffusion, J. Nucl. Mater. 120 (1984) 55 .

[78] A. B. Sivak, P. A. Sivak, V. A. Romanov, V. M. Chernov, Effect of external stresses on efficiency of dislocation sinks in $\mathrm{BCC}(\mathrm{Fe}, \mathrm{V})$ and $\mathrm{FCC}(\mathrm{Cu})$ crystals, Inorg. Mater. Appl. Res. 6 (2015) 466472.

[79] A. B. Sivak, P. A. Sivak, V. A. Romanov, V. M. Chernov, Dislocation sinks efficiency for self-point defects in iron and vanadium crystals, Inorg. Mater. Appl. Res. 6 (2015) 105113. 
[80] W. G. Wolfer, M. Ashkin, Stress-induced diffusion of point defects to spherical sinks, J. Appl. Phys. 46 (1975) 547. 